\title{
Bosonic higher spin gravity in any dimension with dynamical two-form
}

\author{
Cesar Arias, ${ }^{a}$ Roberto Bonezzi ${ }^{b}$ and Per Sundell ${ }^{a}$ \\ ${ }^{a}$ Departamento de Ciencias Físicas, Universidad Andres Bello, \\ Sazié 2212, Piso 7, Santiago de Chile, Chile \\ ${ }^{b}$ Institute for Physics, Humboldt University Berlin, \\ Zum Großen Windkanal 6, D-12489 Berlin, Germany \\ E-mail: cesar.arias@unab.cl, bonezzi@physik.hu-berlin.de, \\ per.sundell@unab.cl
}

ABSTRACT: We alter Vasiliev's original bosonic higher spin gravity in any dimension beyond the linearized level by factoring out a modified $s p(2)$ gauge algebra. The new model can be embedded together with a dynamical two-form and an extra dynamical one-form into a flat Quillen superconnection. Further duality and $s p(2)$ ghost extensions lead to a FrobeniusChern-Simons action in which the $s p(2)$ and higher spin gauge symmetries are subsumed into a unified Cartan gauge group at the fully non-linear level.

Keywords: Higher Spin Gravity, Non-Commutative Geometry, String Field Theory

ArXiv EPrint: 1712.03135 


\section{Contents}

1 Introduction 1

2 Vasiliev's Type A model 3

2.1 Master field equations 3

2.2 Diagonal $s p(2)$ generators 6

$\begin{array}{lll}2.3 & \text { Star product, boundary conditions and } s p(2) \text { symmetry } & 7\end{array}$

3 New Type A model 9

3.1 Alternative $s p(2)$ gauging $\quad 9$

$\begin{array}{lll}3.2 & \text { Perturbative solution in integrable gauge } & 10\end{array}$

3.3 Gauge function for asymptotically anti-de Sitter solutions 11

3.4 Linearized gauge function and Central On Mass Shell Theorem 13

$\begin{array}{lll}3.5 & s p(2) \text { gauging } & 15\end{array}$

$\begin{array}{lll}3.5 .1 & \text { Imposing } s p(2) \text { invariance } & 15\end{array}$

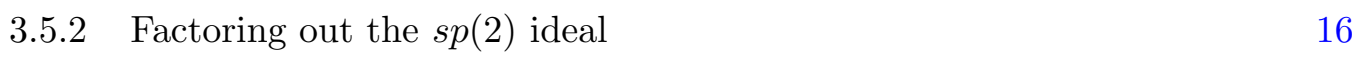

4 Frobenius-Chern-Simons extension $\quad 18$

$\begin{array}{lll}4.1 & \text { Dynamical two-form } & 18\end{array}$

4.2 Superconnection 21

$\begin{array}{ll}4.3 & \text { Action with dynamical two-form } \\ & 22\end{array}$

5 Conclusions $\quad 26$

$\begin{array}{ll}\text { A Ghost algebra } & 27\end{array}$

$\begin{array}{ll}\text { B Trace operations on graded associative algebras } & 30\end{array}$

\section{Introduction}

Higher spin gravities are extensions of ordinary gravity by Fronsdal fields governed by nonabelian higher spin gauge symmetries. The resulting higher spin geometries consist of noncommutative symplectic manifolds given by (symplectic) twistor spaces fibered over phase-spacetimes, as described by Cartan integrable systems found by Vasiliev, first in four and lower spacetime dimensions [1-3] using spinorial twistors, and later in any spacetime dimension [4] using vectorial twistors and $s p(2)$ gauge symmetries; for reviews, see [5-8].

In this paper, we shall be mainly concerned with the vectorial models in any dimension. These consist perturbatively of one real Fronsdal field for every integer spin, including a parity even scalar field. The fields with odd spin can be consistently set to zero, leading to a vectorial version in any dimension of the four-dimensional minimal bosonic spinorial Type 
A model [9]. ${ }^{1}$ In what follows, we shall first provide an alternative vectorial Type A model by modifying the $s p(2)$ gauging without affecting the higher spin gauge algebra nor the perturbative spectrum. We then extend its field content by a dynamical two-form and an extra dynamical one-form so as to obtain a vectorial generalization of the four-dimensional spinorial Frobenius-Chern-Simons model (FCS) proposed in [10]. The FCS model contains the alternative Type A model as a consistent truncation. At the linearized level, the FCS model contains additional degrees of freedom arising from non-trivial cohomology elements in the dynamical two-form. In this paper, we shall restrict, however, the perturbative analysis to the alternative Type A model.

The FCS model is more predictive than its Type A model truncations, as it possesses an enlarged bi-fundamental gauge group that drastically reduces the number of higher spin gauge invariants, and facilitates an off-shell formulation using topological field theory methods leading to an on-shell action with only a finite number of free parameters. Moreover, the FCS model, which is formulated in terms of a Quillen superconnection [38] valued in a Frobenius algebra, is akin to a topological open string field theory [11-13]. Topological strings provide a natural framework for coupling massless higher spin particles to ordinary tensionless strings in anti-de Sitter backgrounds [14-17] in accordance with holography $[14,18-20]$. Further developments of the FCS model may thus open up new windows to holography, permitting access to a wide range of physically relevant quantum field theories in four and higher dimensions, including four-dimensional pure Yang-Mills theories.

The resulting framework provides a path-integral quantization of higher spin gravity using the language of topological quantum field theories on noncommutative Poisson manifolds $[25,26]$ (see also [10, 27]) which can be used to introduce the notion of star-product locality of equations of motion, covariant Hamiltonian Lagrangians and other densities used for constructing observables [25, 26, 30, 31]. Indeed, as stressed by [22], there is a tension between the quasi-Riemannian notion of spacetime locality and (nonabelian) higher spin gauge symmetry that obstructs the Fronsdal program [24], i.e. the application of the Noether procedure so as to obtain a classical action for deformed Fronsdal fields; for related work on Vasiliev's formulation to the deformed Fronsdal program, see [21, 23]. Thus, it makes more sense to think of the deformed Fronsdal theory, viewed as a stand-alone quantum field theory without any reference to higher dimensional noncommutative geometry, as a quantum effective theory governed by higher spin gauge symmetry and unitarity without any classical limit directly on spacetime.

In the topological field theory realization of higher spin gravity, the physical states arise as boundary states of a topological bulk theory created by generalized Chern classes [10]. A subset of these do not receive any quantum corrections, mainly due to the conservation of form degrees at bulk vertices. On-shell, they are related to zero-form charges [29, 31] which indeed serve as generating functionals for holographic correlation functions [30, 32, 33]; for more recent progress, see [34]. Accordingly, the FCS model subjected to appropriate boundary conditions should contain a Vasiliev branch that is equivalent on-shell to the quantum effective deformed Fronsdal theory. Of key importance in this approach is the

\footnotetext{
${ }^{1}$ Strictly speaking, the equivalence between the spinorial and vectorial Type A models in four dimensions remains to be established beyond the linearized level.
} 
fact that the original Vasiliev system contains closed and central elements in form degree two, which combine with the Weyl zero-form into deformations of the noncommutative higher spin geometry. In the FCS model, these elements arise as particular background values of a dynamical two-form master field, which one may thus think of as points in a larger moduli space of noncommutative geometries [10]. The resulting FCS landscape may thus contain new bridges between holographically dual field theories and first- and second-quantized topological field theories; indeed, similar correspondences exist in string and M-theory $[35,36]$.

In the vectorial FCS model, the presence of the dynamical two-form implies that the equations of motion cannot be rewritten as a Wigner deformed oscillator algebra on a general background. ${ }^{2}$ In Vasiliev's original model, these oscillators are used to define an $s p(2)$ algebra factored out from the twistor space on-shell. In order to introduce the twoform consistently, we instead factor out an alternative $s p(2)$ algebra, that does not refer to any underlying Wigner deformed oscillator algebra. We emphasize that the existence of two possible $s p(2)$ gaugings stems from the fact that both meet the basic criteria for choosing the $s p(2)$ gauge algebra, namely Cartan integrability of the full nonlinear system, and Vasiliev's Central On Mass Shell Theorem [5], i.e. consistency of the linearized system, as we shall spell out in detail in section 3 . Thus, starting at the linearized level, where the two theories are clearly equivalent, the old gauging is possible only on special noncommutative manifolds while the new gauging, which is thus more akin to topological open string theory, is distinguished by its potential extension to general noncommutative manifolds.

The paper is organized as follows: in section 2, we review Vasiliev's original Type A model. In section 3, we proceed with the formulation of the new Type A model based on a modified $s p(2)$ gauging. We compare the new model with the original model at the (full) perturbative level as well as at the level of higher spin invariants. In section 4, we couple the new model to a dynamical two-form and further extend the system to a flat Quillen superconnection. Introducing $s p(2)$ ghosts, we construct a BRST operator and construct an action that makes the Quillen flatness condition and $s p(2)$ gauge conditions variational; the formalism also provides a fully non-linear extension of the $s p(2)$ gauge symmetries compatible with Cartan integrability. We conclude in section 5 pointing to a number of future directions.

\section{Vasiliev's Type A model}

In this section, we outline Vasiliev's original formulation of self-interacting totally symmetric higher spin gauge fields in arbitrary spacetime dimensions.

\subsection{Master field equations}

Vasiliev's higher spin gravity is formulated in terms of horizontal differential forms on noncommutative fibered spaces, which we refer to as correspondence spaces. These forms

\footnotetext{
${ }^{2}$ In the four-dimensional spinorial FCS theory with spinorial twistor space, this implies that the Lorentz covariance can only be made manifest on its Vasiliev branch, as here the deformed oscillator algebra is restored.
} 
belong to a differential graded associative algebra with compatible differential $d(\cdot)$ and binary product $(\cdot) \star(\cdot)$. Locally, the correspondence space is a direct product of a base with coordinates $\left(X^{M}, Z_{i}^{A}\right)$ and a fiber with coordinates $Y_{i}^{A}$, where $X^{M}$ coordinatize a commutative manifold containing spacetime, and $Z_{i}^{A}$ and $Y_{i}^{A}$ are noncommutative coordinates with non-trivial commutation relations

$$
\left[Y_{i}^{A}, Y_{j}^{B}\right]_{\star}=2 i \epsilon_{i j} \eta^{A B}, \quad\left[Z_{i}^{A}, Z_{j}^{B}\right]_{\star}=-2 i \epsilon_{i j} \eta^{A B}
$$

introducing the $s o(2, D-1)$ invariant tensor $\eta^{A B}$ and the $s p(2)$ invariant tensor $\epsilon_{i j}$. Lorentz tensors are defined by a constant frame field $\left(V_{A}, V_{A}^{a}\right)$ obeying $\eta^{A B} V_{A} V_{B}=-1, \eta^{A B} V_{A}^{a} V_{B}=0$ and $\eta^{A B} V_{A}^{a} V_{B}^{b}=\eta^{a b}$; one also defines $Y_{i}:=V_{A} Y_{i}^{A}$ and $Y_{i}^{a}=V_{A}^{a} Y_{i}^{A}$ idem $Z_{i}$ and $Z_{i}^{a}$. Locally, the horizontal projection of the differential on the correspondence spaces is given by

$$
d=d X^{M} \partial_{M}+d Z_{i}^{A} \frac{\partial}{\partial Z_{i}^{A}}
$$

where $\left(d X^{M}, d Z_{i}^{A}\right)$ are anti-commuting line elements (that star commute with the coordinates).

The dynamical fields, all of which are horizontal, are a twisted-adjoint zero-form $\Phi(X, Z ; Y)$ and an adjoint one-form $W=d X^{M} W_{M}(X, Z ; Y)+d Z^{A i} W_{A i}(X, Z ; Y)$, which we shall refer to as master fields as they comprise infinite towers of tensor fields on the commuting manifold. The system is put on-shell by i) imposing the constraints

$$
\begin{aligned}
F+\Phi \star J & =0, & D \Phi & =0, \\
D K_{i j} & =0, & {\left[K_{i j}, \Phi\right]_{\pi} } & =0,
\end{aligned}
$$

where $K_{i j}$ generate an $s p(2)$ algebra, viz.

$$
\left[K_{i j}, K_{k l}\right]_{\star}=4 i \epsilon_{(j \mid(k} K_{l) \mid i)},
$$

which together form a quasi-free differential algebra; and ii) factoring out the orbits generated by the shift transformations

$$
\delta W=K_{i j} \star \alpha^{i j}, \quad \delta \Phi=K_{i j} \star \beta^{i j}, \quad \delta K_{i j}=0,
$$

where $\alpha^{i j}$ and $\beta^{i j}$ are triplets under the adjoint and twisted-adjoint action of $s p(2)$, respectively, viz.

$$
\left[K_{i j}, \alpha^{k l}\right]_{\star}=4 i \delta_{(i}^{(k} \alpha_{j)}^{l)}, \quad\left[K_{i j}, \beta^{k l}\right]_{\pi}=4 i \delta_{(i}^{(k} \beta_{j)}^{l)} .
$$

In the above, the following definitions have been used: the curvature and covariant derivatives

$$
\begin{aligned}
F & :=d W+W \star W, \\
D \Phi & :=d \Phi+[W, \Phi]_{\pi}, \\
D K_{i j} & :=d K_{i j}+\left[W, K_{i j}\right]_{\star},
\end{aligned}
$$


where the $\pi$-twisted commutator

$$
[f, g]_{\pi}:=f \star g-(-1)^{\operatorname{deg}(f) \operatorname{deg}(g)} g \star \pi(f),
$$

using the automorphism $\pi$ of the star product algebra defined by

$$
\pi\left(X^{M}, Z_{i}^{a}, Z_{i} ; Y_{i}^{a}, Y_{i}\right):=\left(X^{M}, Z_{i}^{a},-Z_{i} ; Y_{i}^{a},-Y_{i}\right), \quad \pi d=d \pi .
$$

The element $J$ is the closed two-form

$$
J=-\frac{i}{4} d Z^{i} d Z_{i} \kappa
$$

where $\kappa$ is an inner Klein operator obeying

$$
d Z^{i} d Z_{i}(\kappa \star f-\pi(f) \star \kappa)=0, \quad \kappa \star \kappa=1,
$$

for general horizontal forms $f$. It follows that

$$
\kappa=\kappa_{Y} \star \kappa_{Z},
$$

where

$$
d Z^{i} d Z_{i}\left(\kappa_{Z} \star f-\pi_{Z}(f) \star \kappa_{Z}\right)=0, \quad \kappa_{Y} \star f-\pi_{Y}(f) \star \kappa_{Y}=0
$$

for general horizontal forms, and

$$
\begin{aligned}
& \pi_{Z}\left(X^{M}, Z_{i}^{a}, Z_{i} ; Y_{i}^{a}, Y_{i}\right):=\left(X^{M}, Z_{i}^{a},-Z_{i} ; Y_{i}^{a}, Y_{i}\right), \quad \pi_{Z} d=d \pi_{Z}, \\
& \pi_{Y}\left(X^{M}, Z_{i}^{a}, Z_{i} ; Y_{i}^{a}, Y_{i}\right):=\left(X^{M}, Z_{i}^{a}, Z_{i} ; Y_{i}^{a},-Y_{i}\right) .
\end{aligned}
$$

Finally, the master fields obey the reality conditions

$$
W^{\dagger}=-W, \quad \Phi^{\dagger}=\pi(\Phi), \quad J^{\dagger}=-J,
$$

where the hermitian conjugation operation is defined by

$$
\begin{aligned}
(d f)^{\dagger} & =d\left(f^{\dagger}\right), \quad(f \star g)^{\dagger}=(-1)^{\operatorname{deg}(f) \operatorname{deg}(g)} g^{\dagger} \star f^{\dagger}, \\
\left(X^{M}, Y_{i}^{A}, Z_{i}^{A}\right)^{\dagger} & =\left(X^{M}, Y_{i}^{A},-Z_{i}^{A}\right) .
\end{aligned}
$$

The equations of motion define a Cartan integrable system with Cartan gauge transformations

$$
\delta_{\epsilon} W=D \epsilon, \quad \delta_{\epsilon} \Phi=-[\epsilon, \Phi]_{\pi}, \quad \delta_{\epsilon} K_{i j}=-\left[\epsilon, K_{i j}\right]_{\star} .
$$

The shift transformations (2.6), which transform the field equations proportionally to $K_{i j}$, can be turned into Cartan gauge symmetries of an extended system, as will be spelled out in section 4 . 


\subsection{Diagonal $\operatorname{sp}(2)$ generators}

In Vasiliev's model, the $s p(2)$ gauge algebra is generated by

$$
K_{i j}^{(\mathrm{diag})}:=K_{i j}^{(0)}-K_{i j}^{(S)}, \quad K_{i j}^{(0)}:=K_{i j}^{(Y)}+K_{i j}^{(Z)},
$$

where the two first generators are field independent, viz.

$$
K_{i j}^{(Y)}:=\frac{1}{2} Y_{(i}^{A} \star Y_{j) A} \equiv K_{i j}, \quad K_{i j}^{(Z)}:=-\frac{1}{2} Z_{(i}^{A} \star Z_{j) A},
$$

and $K_{i j}^{(S)}$ is the field dependent generator

$$
K_{i j}^{(S)}:=-\frac{1}{2} S_{(i}^{A} \star S_{j) A},
$$

built from the generalized Wigner deformed oscillator

$$
S_{A i}:=Z_{A i}-2 i W_{A i}, \quad\left(S_{A i}\right)^{\dagger}=-S_{A i},
$$

which is an adjoint element in the sense that

$$
\delta_{\epsilon} S_{A i}=-\left[\epsilon, S_{A i}\right]_{\star} .
$$

The $s p(2)$ generators defined above form three copies of $s p(2)$, viz.

$$
\begin{aligned}
{\left[K_{i j}^{(Y)}, K_{k l}^{(Y)}\right]_{\star} } & =4 i \epsilon_{(j \mid(k} K_{l) \mid i)}^{(Y)}, \quad\left[K_{i j}^{(Z)}, K_{k l}^{(Z)}\right]_{\star}=4 i \epsilon_{(j \mid(k} K_{l) \mid i)}^{(Z)}, \\
{\left[K_{i j}^{(S)}, K_{k l}^{(S)}\right]_{\star} } & =4 i \epsilon_{(j \mid(k} K_{l) \mid i)}^{(S)} .
\end{aligned}
$$

The last relation follows from

$$
\begin{aligned}
{\left[S_{A i}, S_{B j}\right]_{\star} } & =-2 i \epsilon_{i j}\left(\eta_{A B}-V_{A} V_{B} \Phi \star \kappa\right), \\
S_{a i} \star \Phi-\Phi \star \pi\left(S_{a i}\right) & =0, \quad S_{i} \star \Phi+\Phi \star \pi\left(S_{i}\right)=0,
\end{aligned}
$$

which is an equivalent way of writing $F_{A i, B j}=-\frac{i}{2} \epsilon_{i j} V_{A} V_{B} \Phi \star \kappa$ and $D_{A i} \Phi=0$ using an undeformed oscillator $S_{a i}$ and a deformed oscillator $S_{i}:=V^{A} S_{A i}$, with $\Phi$ playing the role of Wigner deformation parameter.

As for the $s p(2)$ invariance conditions, it follows from $D_{M} S_{A i}=0$ and $\left[S_{A i}, \Phi\right]_{\pi}=0$ that

$$
\begin{aligned}
D_{M} K_{i j}^{(\text {diag })} & =0 \Leftrightarrow\left[K_{i j}^{(0)}, W_{M}\right]_{\star}=0, \\
{\left[K_{i j}^{(\text {diag })}, \Phi\right]_{\pi} } & =0 \Leftrightarrow\left[K_{i j}^{(0)}, \Phi\right]_{\star}=0,
\end{aligned}
$$

while

$$
D_{A i} K_{j k}^{(\mathrm{diag})}=0 \quad \Leftrightarrow \quad\left[S_{A i}, K_{j k}^{(0)}-K_{j k}^{(S)}\right]_{\star}=0 \quad \Leftrightarrow \quad\left[K_{i j}^{(0)}, S_{A k}\right]_{\star}=2 i S_{A(i} \epsilon_{j) k},
$$

from which it follows that

$$
\begin{aligned}
{\left[K_{i j}^{(\mathrm{diag})}, K_{k l}^{(\mathrm{diag})}\right]_{\star} } & =4 i \epsilon_{(j \mid(k}\left(K_{l) \mid i(}^{(0)}+K_{l) \mid i)}^{(S)}\right)-\left[K_{i j}^{(0)}, K_{k l}^{(S)}\right]_{\star}-\left[K_{i j}^{(S)}, K_{k l}^{(0)}\right]_{\star} \\
& =4 i \epsilon_{(j \mid(k}\left(K_{l) \mid i)}^{(0)}-K_{l) \mid i)}^{(S)}\right)=4 i \epsilon_{(j \mid(k} K_{l) \mid i)}^{(\mathrm{diag})},
\end{aligned}
$$


i.e. the desired $s p(2)$ commutation rules (2.5). Under a Cartan gauge transformation, one has

$$
\delta_{\epsilon} K_{i j}^{(\operatorname{diag})}=-\delta_{\epsilon} K_{i j}^{(S)}=-\left[\epsilon, K_{i j}^{(S)}\right],
$$

and hence $\delta_{\epsilon} K_{i j}^{(\mathrm{diag})}=-\left[\epsilon, K_{i j}^{(\mathrm{diag})}\right]_{\star}$ holds true provided that

$$
\left[K_{i j}^{(0)}, \epsilon\right]_{\star}=0,
$$

which is indeed compatible with (2.29).

\subsection{Star product, boundary conditions and $s p(2)$ symmetry}

Although there does not exist any canonical realization of the star product algebra in terms of symbols, there are two choices of operator ordering that are particularly convenient for imposing boundary conditions on correspondence space and constructing classical solution spaces.

We recall that a given operator ordering amounts to a Wigner map that realizes the star product as a non-local composition rule for symbols given by a twisted convolution formula, and that different ordering schemes are connected by formal Kontsevich gauge transformations which can be thought of as changes of bases for the operator algebra generated by symmetric poly-vector fields acting on the space of symbols, including the ordinary correspondence space bundle maps, i.e. higher spin gauge transformations and symplectomorphisms of the base manifold. The boundary conditions thus serve two dual purposes:

i) the selection of a class of horizontal forms with finite globally defined integrals over cycles in correspondence space; and

ii) the identification of a basis for deformed Fronsdal fields in weakly coupled regions of spacetime.

The finite cycles, which play the role of observables, are left invariant by a subgroup of the bundle maps, usually referred to as the small gauge transformations. The remaining coset consisting of gauge functions, or large gauge transformations, act on the space of zero-form integration constants (and other fluxes in higher form degree when present) to produce orbits making up a classical moduli space. The higher spin amplitudes can then be computed by relating the classical moduli to polarization data for deformed Fronsdal fields in asymptotically anti-de Sitter regions and identifying the on-shell action.

In order to implement (i), it is convenient to use the Weyl order defined by

$$
[f \star g]_{\mathrm{w}}(Y, Z)=\int d \mu d \tilde{\mu} e^{i\left(V_{A}^{i} U_{i}^{A}+\tilde{V}_{A}^{i} \tilde{U}_{i}^{A}\right)}[f]_{\mathrm{w}}(Y+U, Z+\tilde{U})[g]_{\mathrm{w}}(Y+V, Z-\tilde{V}),
$$

where $[\cdot]_{\text {w }}$ denotes the corresponding Wigner map and $d \mu=(2 \pi)^{-2(D+1)} d^{2(D+1)} U d^{2(D+1)} V$ $i d e m d \tilde{\mu}$. This order exhibits the fibration of the correspondence space in the sense that expanding horizontal forms over complete sets of fiber functions $T_{\lambda}(Y)$ multiplied by mode forms $f^{\lambda}(X, Z ; d X, d Z)$ on the base manifold, viz.

$$
f=\sum_{\lambda} T_{\lambda} \star f^{\lambda}
$$


one has

$$
[f]_{\mathrm{w}}=\sum_{\lambda}\left[T_{\lambda}\right]_{\mathrm{w}}\left[f^{\lambda}\right]_{\mathrm{w}}
$$

Thus, the star product algebras in the fiber and on the base are separated from each other, which facilitates the construction of higher spin invariants as well as classical moduli spaces. In particular, letting [.] ${ }^{\mathrm{w}}$ denote the inverse Wigner map in Weyl order, the inner Klein operators in (2.15) are given by

$$
\kappa_{Y}=\left[2 \pi \delta^{2}\left(Y_{i}\right)\right]^{\mathrm{w}}, \quad \kappa_{Z}=\left[2 \pi \delta^{2}\left(Z_{i}\right)\right]^{\mathrm{w}}, \quad \kappa=\left[(2 \pi)^{2} \delta^{2}\left(Y_{i}\right) \delta^{2}\left(Z_{i}\right)\right]^{\mathrm{w}},
$$

enabling obtaining $\left(T_{\lambda}, f^{\lambda}\right)$ perturbatively as distributions.

The implementation of (ii), on the other hand, requires an ordering scheme in which the master fields are real analytic in $Y$ at $Y=Z=0$, as the corresponding Taylor coefficients define the unfolded deformed Fronsdal fields and their Weyl curvatures. This can be achieved by going to normal order in which

$$
[f \star g]_{\mathrm{n}}(Y, Z)=\int d \mu e^{i V_{A}^{i} U_{i}^{A}}[f]_{\mathrm{n}}(Y+U, Z+U)[g]_{\mathrm{n}}(Y+V, Z-V),
$$

where $[\cdot]_{\mathrm{n}}$ denotes the corresponding Wigner map. Consequently, the expansion (2.36) is equivalent to

$$
[f]_{\mathrm{n}}(Y, Z)=\sum_{\lambda} \int d \mu e^{i V_{A}^{i} U_{i}^{A}}\left[T_{\lambda}\right]_{\mathrm{w}}(Y+U)\left[f^{\lambda}\right]_{\mathrm{w}}(Z-V),
$$

as $\left[T_{\lambda}\right]_{\mathrm{n}}=\left[T_{\lambda}\right]_{\mathrm{w}}$ and $\left[f^{\lambda}\right]_{\mathrm{n}}=\left[f^{\lambda}\right]_{\mathrm{w}}$, which makes the symbols in normal order less singular than those in Weyl order. In particular,

$$
\kappa_{Y}=\left[2 \pi \delta^{2}\left(Y_{i}\right)\right]^{\mathrm{n}}, \quad \kappa_{Z}=\left[2 \pi \delta^{2}\left(Z_{i}\right)\right]^{\mathrm{n}}, \quad \kappa=\left[\exp \left(i Y^{i} Z_{i}\right)\right]^{\mathrm{n}},
$$

where $[\cdot]^{\mathrm{n}}$ denotes the inverse Wigner map in normal order.

The unfolded description of free Fronsdal fields, as spelled out by the Central On Mass Shell Theorem [5], is contained in the linearization of [4]

$$
\left.\left[F_{M N}\right]_{\mathrm{n}}\right|_{Z=0}=0,\left.\quad\left[D_{M} \Phi\right]_{\mathrm{n}}\right|_{Z=0}=0
$$

around the anti-de Sitter background for $W$, provided that

a) all linearized master fields have symbols in normal order that are real analytic in $Y$ at $Y=Z=0$; and

b) the gauge condition

$$
W_{a i}=0, \quad Z^{i}\left[W_{i}\right]_{\mathrm{n}}=0,
$$

which we shall refer to as the Vasiliev gauge, holds in the linearized approximation. 
Thus, asymptotically anti-de Sitter regions containing deformed Fronsdal fields arise by imposing $(a)$ and $(b)$ in the leading order of the generalized Fefferman-Graham expansion in the radial coordinate to all orders in classical perturbation theory, and simultaneously imposing (i), which provides a "dual" boundary condition on the gauge functions at infinity of $\mathcal{Z}$.

Finally, turning to the rationale behind the choice of the $s p(2)^{(\mathrm{diag})}$ generators made in (2.22), it consists of the facts that:

I) it amounts to gauging the simultaneous transformation of the doublets $\left(Y_{i}^{A}, Z_{i}^{A}, d Z_{i}^{A}\right.$, $W_{A}^{i}$ ) which is a manifest symmetry in normal order (where $\kappa_{\mathrm{n}}$ is given by $(2.41)$ ); and

II) in the leading order of the perturbative expansion around the anti-de Sitter vacuum, the $s p(2)^{(\mathrm{diag})}$ generators reduce to those of $s p(2)^{(Y)}$, whose factoring out from the linearized fluctuations in $W_{M}$ and $\Phi$ yields unfolded free Fronsdal tensors and corresponding Weyl tensors on-shell, respectively.

However, as we shall exploit next, property (II), which refers explicitly to the Wigner deformed oscillator algebra, is not necessary for consistency, and can hence be relaxed so as to couple the system to a dynamical two-form master field.

\section{New Type A model}

In this section, we define the new Type A model and demonstrate its perturbative consistency including its abiding by the requirements of the Central On Mass Shell Theorem at the linearized level.

\subsection{Alternative $s p(2)$ gauging}

The consistency of the $s p(2)$ gauging of Vasiliev's original Type A model relies on the facts that the $s p(2)$ generators

i) form a star product Lie algebra which ensures consistency of the classical perturbation theory without any additional algebraic constraints on the master fields; and

ii) are given by $s p(2)^{(Y)}$ in the leading order of the perturbative expansion around the anti-de Sitter vacuum which ensures a linearized description in terms of free Fronsdal fields.

As we shall demonstrate in what follows, both conditions hold true if one instead of $K_{i j}^{\text {(diag) }}$ uses

$$
K_{i j}^{(L)}:=L^{-1} \star K_{i j}^{(Y)} \star L,
$$

where $L$ is the gauge function on the commuting factor of the base manifold of the correspondence space. More precisely, taking the fibration of the correspondence space to be

$$
\mathcal{Y} \hookrightarrow \mathcal{C} \rightarrow \mathcal{X} \times \mathcal{Z},
$$


where $\mathcal{C}$ is the total space, $\mathcal{Y}$ the fiber, and $\mathcal{X}$ and $\mathcal{Z}$, respectively, the commuting and symplectic components of its base, the gauge function is given by the path ordered exponential

$$
L=P \exp _{\star} \int_{C_{p ; p_{0}}} W,
$$

where $p, p_{0} \in \mathcal{C} ; C_{p ; p_{0}}$ is a path in $\mathcal{C}$ from $p_{0}$ to $p$ that is transverse to $T^{*} \mathcal{Z}$; and $p_{0}$ sit over a given point $x_{0} \in \mathcal{X}$.

As we shall see, the Vasiliev gauge condition (2.43) is reached in the two Type A models using two different methods for homotopy contracting the de Rham differential $d^{\prime}:=d Z_{i}^{A} \frac{\partial}{\partial Z_{i}^{A}}$ on $\mathcal{Z}$ :

- In the original Type A model, one uses a homotopy contractor on $\mathcal{Z}$ defined in normal order and that commutes to $s p(2)^{(\mathrm{diag})}$;

- In the new Type A model, one instead starts by using a homotopy contractor on $\mathcal{Z}$ defined in Weyl order and that commutes to $s p(2)^{(Y)}$ in an "integrable" gauge where $L=1$, after which one implements eq. (2.43) by first switching on an anti-de Sitter gauge function $L^{(0)}$ that commutes to $s p(2)^{(Y)}$ followed by a linearized gauge function $L^{(1)}$ that does not commute to $s p(2)^{(Y)}$ hence switching on the linearized piece of $s p(2)^{(L)}$.

It follows that the two models coincide at the free level, which essentially amounts to the existence of a linearized gauge function, as will be shown below. To compare the models beyond the free level, we shall propose to use a set of higher spin gauge invariants, referred to as zero-form charges [32], given by traces of adjoint zero-forms on $\mathcal{X}$ including a quasiprojector that annihilates the two-sided ideals generated by the $s p(2)$-generators. Whether these observables can be used to map the two models into each other, remains, however, an open problem. ${ }^{3}$

\subsection{Perturbative solution in integrable gauge}

The gauge function can be used to solve the differential equations in $\mathcal{X}$, viz.

$$
W=L^{-1} \star\left(W^{\prime}+d\right) \star L, \quad \Phi=L^{-1} \star \Phi^{\prime} \star \pi(L), \quad W_{M}^{\prime}=0,
$$

where the primed fields, which are thus $X$-independent, obey the reduced equations

$$
d^{\prime} W^{\prime}+W^{\prime} \star W^{\prime}+\Phi^{\prime} \star J=0, \quad d^{\prime} \Phi^{\prime}+W^{\prime} \star \Phi^{\prime}-\Phi^{\prime} \star \pi\left(W^{\prime}\right)=0 .
$$

Going to Weyl order, imposing the gauge condition

$$
Z^{i}\left[W_{i}^{\prime}\right]_{\mathrm{w}}=0, \quad W_{a i}^{\prime}=0,
$$

defining zero-form initial data

$$
\left[C^{\prime}\right]_{\mathrm{w}}:=\left.\left[\Phi^{\prime}\right]_{\mathrm{w}}\right|_{Z=0},
$$

\footnotetext{
${ }^{3}$ In the four-dimensional spinorial formulation, the corresponding observables have non-trivial perturbative expansions in terms of boundary data defined in weakly coupled asymptotically anti-de Sitter solutions [10].
} 
and assuming that

$$
\left.W_{i}^{\prime}\right|_{C^{\prime}=0}=0,
$$

i.e. that the vacuum configuration in the vectorial twistor space is trivial, the resulting solution space can be written as

$$
\Phi^{\prime}=C^{\prime}, \quad W^{\prime}=\sum_{n \geqslant 1} w_{n} \star\left(C^{\prime} \star \kappa_{Y}\right)^{\star n},
$$

with perturbative corrections grouped into a generating element

$$
w^{\prime}:=\sum_{n \geqslant 1} w_{n} \nu^{n}, \quad w_{n}=d Z^{i} w_{n, i}\left(Z^{j}\right), \quad \nu \in \mathbb{C},
$$

obeying the deformed oscillator problem [3]

$$
d^{\prime} w^{\prime}+w^{\prime} \star w^{\prime}+\nu j^{\prime}=0, \quad j^{\prime}:=-\frac{i}{4} d Z^{i} d Z_{i} \kappa_{Z},
$$

subject to the gauge and boundary conditions

$$
\pi_{Z}\left(w^{\prime}\right)=0, \quad \imath_{Z^{i}} \vec{\partial}_{i} w^{\prime}=0 .
$$

The deformed oscillator problem can be solved by adapting the method for the fourdimensional spinorial Type A model spelled out in [39]. ${ }^{4}$

\subsection{Gauge function for asymptotically anti-de Sitter solutions}

Let us proceed, still in parallel between the old and new models, by finding the gauge function $L$ mapping the integrable gauge to the Vasiliev gauge (2.43), that we shall assume holds to all orders for the sake of simplicity.

To this end, we expand $C^{\prime}$ and $L$ in terms of the linearized zero-form initial data, that we denote by $C^{\prime(1)}$, viz.

$$
C^{\prime}=\sum_{n \geqslant 1} C^{\prime(n)}, \quad L=\sum_{n \geqslant 0} L^{(n)}=L^{(0)} \star H, \quad H=1+\sum_{n \geqslant 1} H^{(n)},
$$

where $L^{(0)}$ is an anti-de Sitter background gauge function, and likewise

$$
\Phi=\sum_{n \geqslant 1} \Phi^{(n)}, \quad W=\sum_{n \geqslant 0} W^{(n)}
$$

where the superscripts refer to the order in classical perturbative expansion in $C^{\prime(1)}$. We assume that upon going to normal order, the symbols $[\Phi]_{\mathrm{n}},[W]_{\mathrm{n}}$ and $[H]_{\mathrm{n}}$ are bounded at $Z=0$, and that

$$
[\omega]_{\mathrm{n}}:=\left.\left.[W]_{\mathrm{n}}\right|_{Z=0} \equiv\left[d x^{\mu} W_{\mu}\right]_{\mathrm{n}}\right|_{Z=0}, \quad[C]_{\mathrm{n}}:=\left.[\Phi]_{\mathrm{n}}\right|_{Z=0},
$$

\footnotetext{
${ }^{4}$ As a result, $w_{i}^{\prime}=Z_{i} w\left(Z^{+} Z^{-}\right)$where $Z^{ \pm}=U_{i}^{ \pm} Z^{i}$ are creation and annihilation operators defined using an auxiliary $\operatorname{sp}(2)$ frame $U_{i}^{ \pm}$on $\mathcal{Z}$, and $w\left(Z^{+} Z^{-}\right)=\int d \tau \exp \left(\tau Z^{+} Z^{-}\right) \tilde{w}(\tau)$ where $\tilde{w}(\tau)$ is built from a confluent hypergeometric function; for further details, including the decomposition of the moduli space into cells separated by critical Wigner deformation parameters and the incorporation of flat connections on $\mathcal{Z}$, see $[29,39,40]$.
} 
are real analytic at $Y=0$, serving as generating functions for unfolded deformed Fronsdal fields and their Weyl curvatures. Moreover, we assume that $\left[d Z^{i} W_{i}\right]_{\mathrm{n}}$ and $[H]_{\mathrm{n}}$ belong to the space of forms on $\mathcal{Z}$ that are real analytic at $Z=0$. An element $f^{\prime}$ in this space admits the decomposition

$$
f^{\prime}=\rho^{\prime} d^{\prime} f^{\prime}+d^{\prime} g^{\prime}+\delta_{0, \operatorname{deg}\left(f^{\prime}\right)}\left[\left.\left[f^{\prime}\right]_{\mathrm{n}}\right|_{Z=0}\right]^{\mathrm{n}},
$$

locally at $Z=0$, where the homotopy contractor

$$
\rho^{\prime}\left(f^{\prime}\right):=\left[\imath_{\vec{v}^{\prime}}\left(\mathcal{L}_{\vec{v}^{\prime}}\right)^{-1}\left[f^{\prime}\right]_{\mathrm{n}}\right]^{\mathrm{n}}, \quad \vec{v}^{\prime}=Z^{A i} \vec{\partial}_{A i}^{(Z)},
$$

and the inverse Lie derivative admits the integral representation

$$
\mathcal{L}_{\vec{v}^{\prime}}^{-1}=\int_{0}^{1} \frac{d t}{t} t^{\mathcal{L}_{\vec{v}^{\prime}}}
$$

Thus, iterating (2.43) yields [30]

$$
\begin{aligned}
H^{(n)}=H_{0}^{(n)} & -\sum_{n_{1}+n_{2}+n_{3}=n} \rho^{\prime}\left(\left(L^{-1}\right)^{\left(n_{1}\right)} \star W^{\prime\left(n_{2}\right)} \star L^{\left(n_{3}\right)}\right) \\
& -\sum_{n_{1}+n_{2}=n} \rho^{\prime}\left(\left(L^{-1}-\left(L^{(0)}\right)^{-1}\right)^{\left(n_{1}\right)} \star d^{\prime} L^{\left(n_{2}\right)}\right),
\end{aligned}
$$

for $n \geqslant 1$, where

$$
H_{0}^{(n)}:=\left[\left.\left[H^{(n)}\right]_{\mathrm{n}}\right|_{Z=0}\right]^{\mathrm{n}} .
$$

The quantities $H_{0}^{(n)}$ and $C^{\prime(n)}$, which are integration constants on $\mathcal{Z}$ that were omitted in [30], are determined, respectively, by the regularity requirements on $\omega$ and $C$ at $Y=0$ up to a pure gauge term in $H_{0}^{(n)}$, i.e. a piece that is real analytic at $Y=0$, and trivial re-definitions of $C^{\prime(1)}$.

Eq. (3.19) can be obtained starting from

$$
0=\rho^{\prime} W=\rho^{\prime}\left(L^{-1} \star\left(W^{\prime}+d^{\prime}\right) \star L\right),
$$

which follows from (2.43), and rewriting the second term using

$$
L^{-1} \star d^{\prime} L=d^{\prime}\left(\left(L^{(0)}\right)^{-1} \star L\right)+\left(L^{-1}-\left(L^{(0)}\right)^{-1}\right) \star d^{\prime} L,
$$

and

$$
\rho^{\prime} d^{\prime}\left(\left(L^{(0)}\right)^{-1} \star L\right)=H-\left[\left.[H]_{\mathrm{n}}\right|_{Z=0}\right]^{\mathrm{n}} .
$$

Thus

$$
H=\left[\left.[H]_{\mathrm{n}}\right|_{Z=0}\right]^{\mathrm{n}}-\rho^{\prime}\left[L^{-1} \star W^{\prime} \star L+\left(L^{-1}-\left(L^{(0)}\right)^{-1}\right) \star d^{\prime} L\right],
$$

which yields (3.19) upon perturbative expansion.

We recall that (2.43), and hence (3.19), holds only as a boundary condition in $\mathcal{X}$ for asymptotically anti-de Sitter solutions (so as to ensure the existence of asymptotically defined unfolded deformed Fronsdal fields). Inside $\mathcal{X}$ and $\mathcal{Z}$, the equivalence class of gauge functions represented by $L$ is determined by the requirement of finite higher spin invariants. We would also like to remark that $H$ may be non-regular at $Y=0=Z$, which means that the regularity conditions on $\Phi$ at $Y=0=Z$ triggers higher order corrections $C^{\prime(n)}$ $(n \geqslant 2)$ to $C^{\prime}$. 


\subsection{Linearized gauge function and Central On Mass Shell Theorem}

At the linearized level, we have

$$
W^{(1)}=W^{(L, 1)}+\left(D^{(0)}+d^{\prime}\right) H^{(1)}, \quad H^{(1)}=H_{0}^{(1)}-\rho^{\prime} W^{(L, 1)},
$$

where

$$
W^{(L, 1)}:=\left(L^{(0)}\right)^{-1} \star W^{(1)} \star L^{(0)},
$$

which is thus assumed to be bounded at $Z=0$; the components $W_{i}^{(L, 1)}$ are in addition assumed to be real analytic at $Z=0$. It follows that

$$
\omega^{(1)} \equiv\left[\left.\left[W^{(1)}\right]_{\mathrm{n}}\right|_{Z=0}\right]^{\mathrm{n}}=D^{(0)} H_{0}^{(1)}+P^{(1)},
$$

where

$$
\left[P^{(1)}\right]_{\mathrm{n}}:=-\left.\left[D^{(0)} \rho^{\prime} W^{(L, 1)}\right]_{\mathrm{n}}\right|_{Z=0}=-\left.\frac{i}{2} e^{(0) a} \partial_{a}^{(Y) i}\left[W_{i}^{(L, 1)}\right]_{\mathrm{n}}\right|_{Z=0},
$$

obeys [37]

$$
D^{(0)} \omega^{(1)}+e^{(0) a} \wedge e^{(0) b}\left[\partial_{a}^{(Y) i} \partial_{b i}^{(Y)}\left[\left.\left[\Phi^{(1)}\right]_{\mathrm{n}}\right|_{Y_{i}=0}\right]^{\mathrm{n}}=0\right.
$$

where $\left.\left[\Phi^{(1)}\right]_{\mathrm{n}}\right|_{Y_{i}=0}$ is the generating function of higher spin generalized Weyl tensors. Eq. (3.29) consists of a piece that is real analytic at $Y=0$, comprising the central onshell theorem, and a piece that is singular at $Y=0$, that serves to determine $H_{0}^{(1)}$. More precisely, decomposing

$$
P^{(1)}=P_{\text {reg }}^{(1)}+P_{\text {sing }}^{(1)}
$$

where $\left[P_{\text {reg }}^{(1)}\right]_{\mathrm{n}}$ and $\left[P_{\text {sing }}^{(1)}\right]_{\mathrm{n}}$ belong to classes of functions on $\mathcal{Y}$ that are real analytic and non-real analytic at $Y=0$, respectively, for generic spacetime points. Moreover, assuming that $D^{(0)}$ does not mix these classes, ${ }^{5}$ it follows that

$$
D^{(0)} P_{\text {sing }}^{(1)}=0
$$

and hence

$$
P_{\text {sing }}^{(1)}=D^{(0)} Q_{\text {sing }}^{(1)}
$$

Thus, taking

$$
H_{0}^{(1)}=-Q_{\text {sing }}^{(1)},
$$

yields an $\left[\omega^{(1)}\right]_{\mathrm{n}}$ that is real analytic at $Y=0$, that is, a generating function for unfolded Fronsdal fields on-shell. As a by-product, one has the following explicit formula for the expansion in terms of mode functions corresponding to the higher spin representations used in $\left(C^{\prime} \star \kappa_{y}\right)$ :

$$
\left[\omega^{(1)}\right]_{\mathrm{n}}=\left(\left.e^{(0) a} \partial_{a}^{(Y) i}\left[W_{i}^{(L, 1)}\right]_{\mathrm{n}}\right|_{Z=0}\right)_{\mathrm{reg}} .
$$

A few remarks are in order:

\footnotetext{
${ }^{5}$ For example, this is the case if $Y^{A i} \partial_{A i}^{(Y)}$ can be diagonalized in the space of functions on $\mathcal{Y}$.
} 
The analogous construction of $H^{(1)}$ and $\omega^{(1)}$ in the four-dimensional spinorial Type A model has been studied in more detail for zero-form initial data $\Phi^{\prime}$ corresponding to generalized Petrov Type D modes [39, 49], particle modes [51], and solutions with six unbroken anti-de Sitter Killing symmetries [37].

The gauge function method requires working with a class of distributions on $\mathcal{Z} \times \mathcal{Y}$ containing elements on which commutators of partial derivatives in $Y$ and $Z$ do not commute but rather give rise to delta functions at $Z=0$ and $Y=0$. This class consists of elements given by auxiliary integrals of Gaussian kernels, referred to as regular presentations. This presentation, which was first introduced in [39], not only facilitates computations but also ensures associativity as well as the aforementioned property under differentiation with respect to $Y$ and $Z$. In particular, in deriving eq. (3.29), the regular presentation ensures that in calculating $D^{(0)} P^{(1)}$, all partial derivatives in $\mathcal{Y} \times \mathcal{Z}$ actually commute; for a more detailed analysis of this subtlety in the case of the four-dimensional spinorial Type A model, see [52]. Thus, the quantity $D^{(0)} P^{(1)}$ is free from delta functions on $\mathcal{Z} \times \mathcal{Y}$, that is, it can in effect be computed by taking partial derivatives in $Y$ and $Z$ to commute, leading to the appropriate two-form co-cycle in (3.29).

As for the key features of the co-cycle in (3.29), they have been spelled out in the original work of $[1,4]$. Clearly, the co-coycle is real-analytic in $\mathcal{Y}$ provided that $\left[\Phi^{(1)}\right]_{\mathrm{n}}$ is real-analytic in $\mathcal{Y}$. Moreover, the co-cycle is $D^{(0)}$ closed without being locally $D^{(0)}$ trivial in the space of polynomials of finite degree in $\mathcal{Y}$. Thus, (3.29) can be decomposed into an infinite set of equations, one for each Lorentz spin, describing an infinite tower of Fronsdal fields on-shell.

Working, however, with real-analytic powers series in $\mathcal{Y}$, it is possible to Cartan integrate (3.29), i.e. to write the co-cycle as

$$
e^{(0) a} \wedge e^{(0) b}\left[\partial_{a}^{(Y) i} \partial_{b i}^{(Y)}\left[\left.\left[\Phi^{(1)}\right]_{\mathrm{n}}\right|_{Y_{i}=0}\right]^{\mathrm{n}}=-D^{(0)} \omega_{L^{(0)} ; \Phi^{\prime}}^{(1)},\right.
$$

where $\omega_{L^{(0)} ; \Phi^{\prime}}^{(1)}$ is an on-shell construct built from the vacuum gauge function and the (linearized) zero-form integration constant. Thus, on-shell, one has

$$
\omega^{(1)}=\omega_{L^{(0)} ; \Phi^{\prime}}+D^{(0)} \lambda^{(1)}
$$

where $\lambda^{(1)}$ is a linearized gauge function that is real-analytic at $Y=0$; as for an explicit form of $\omega_{L^{(0)} ; \Phi^{\prime}}^{(1)}$ in the four-dimensional Type A model, see [52].

In the above sense, on a specific solution space it is indeed possible to write the linearized co-cycle, which is defined globally at the free level, i.e. it is invariant under abelian gauge transformations, as $D^{(0)}$ of a locally defined potential, viz. $\omega_{L^{(0)} ; \Phi^{\prime}}^{(1)}$. This does not, however, imply that it is possible to remove the co-cycle in eq. (3.29) by a regular field redefinition, as $\omega_{L^{(0)} ; \Phi^{\prime}}^{(1)}$ is a highly non-local construct from the point-of-view of the linearized master fields $\Phi^{(1)}$ and $W_{\mu}^{(1)}$. In this sense, the gauge function method escapes the difficulties pointed out in [53].

Finally, we would like to stress the fact that even if the linearized Weyl zero-form $\left[\Phi^{(1)}\right]_{\mathrm{n}}$ is real-analytic on $\mathcal{Y} \times \mathcal{Z}$ (for generic spacetime points), as is the case for the solution spaces referred to above, the corresponding linearized gauge function $H^{(1)}$ is not real-analytic on 
$\mathcal{Y} \times \mathcal{Z}$ (for any spacetime point). In other words, the analyticity properties in $\mathcal{Y} \times \mathcal{Z}$ of the gauge functions and zero-form integration constants, are quite distinct from those of the linearized master fields $\left[\Phi^{(1)}\right]_{\mathrm{n}}$ and $\omega^{(1)}$; for a more detailed study of boundary conditions in the case of asymptotically locally anti-de Sitter solution spaces to the four-dimensional spinorial Type A model, see [52].

\section{$3.5 s p(2)$ gauging}

In what follows, we first impose the $s p(2)$ invariance conditions and then factor out the $s p(2)$ ideals at the level of higher spin invariants.

\subsubsection{Imposing $\operatorname{sp}(2)$ invariance}

Vasiliev's Type A model. The $s p(2)^{(\mathrm{diag})}$ invariance conditions read

$$
\begin{aligned}
D_{M} K_{i j}^{(\text {diag })}=0 \Leftrightarrow\left[K_{i j}^{(Y)}+K_{i j}^{(Z)}, W_{M}\right]_{\star}=0, \\
D_{A k} K_{i j}^{(\text {diag })}=0 \Leftrightarrow\left[K_{i j}^{(Y)}+K_{i j}^{(Z)}, S_{A k}\right]_{\star}=4 i S_{A(i} \epsilon_{j) k}, \\
{\left[\Phi, K_{i j}^{(\text {diag })}\right]_{\star}=0 \Leftrightarrow\left[K_{i j}^{(Y)}+K_{i j}^{(Z)}, \Phi\right]_{\star}=0 . }
\end{aligned}
$$

In the integrable gauge, these conditions are equivalent to

$$
\left[K_{i j}^{(Y)}, C^{\prime}\right]_{\star}=0
$$

In the Vasiliev gauge, the $s p(2)^{(\mathrm{diag})}$ invariance holds provided that

$$
\left[K_{i j}^{(Y)}+K_{i j}^{(Z)}, L^{(0)}\right]_{\star}=\left[K_{i j}^{(Y)}, L^{(0)}\right]_{\star}=0,
$$

as this condition implies that $\left[K_{i j}^{(Y)}+K_{i j}^{(Z)}, L\right]_{\star}=0$ by virtue of the fact that $\rho^{\prime}$ is $s p(2)^{(\operatorname{diag})}$ invariant.

New Type A model. In the integrable gauge, the $s p(2)^{(L)}$ generators are given by those of $s p(2)^{(Y)}$; hence the $s p(2)^{(L)}$ invariance conditions read

$$
\left[K_{i j}^{(Y)}, W^{\prime}\right]_{\star}=0=\left[K_{i j}^{(Y)}, \Phi^{\prime}\right]_{\star},
$$

which are equivalent to

$$
\left[K_{i j}^{(Y)}, C^{\prime}\right]_{\star}=0 .
$$

We note that, in Weyl order, the basic contraction rules, viz.

$$
\begin{array}{rlrl}
Y_{i}^{A} \star Y_{j}^{B} & :=\left[Y_{i}^{A} Y_{j}^{B}\right]_{\mathrm{w}}+i \eta^{A B} \epsilon_{i j}, & Y_{i}^{A} \star Z_{j}^{B}:=\left[Y_{i}^{A} Z_{j}^{B}\right]_{\mathrm{w}}, \\
Z_{i}^{A} \star Y_{j}^{B}:=\left[Z_{i}^{A} Y_{j}^{B}\right]_{\mathrm{w}}, & Z_{i}^{A} \star Z_{j}^{B}:=\left[Z_{i}^{A} Z_{j}^{B}\right]_{\mathrm{w}}-i \eta^{A B} \epsilon_{i j},
\end{array}
$$

as well as the inner Kleinian

$$
\kappa=\kappa_{Y} \star \kappa_{Z}, \quad \kappa_{Y}=\left[2 \pi \delta^{2}\left(Y^{i}\right)\right]^{\mathrm{w}}, \quad \kappa_{Z}=\left[2 \pi \delta^{2}\left(Z^{i}\right)\right]^{\mathrm{w}},
$$

exhibit manifest $s p(2)^{(Y)} \times s p(2)^{(Z)}$ symmetry. Thus, in Weyl order, both the $\star$ product and the central element $J$ are invariant under $s p(2)^{(Y)} \times s p(2)^{(Z)}$. Hence, the $s p(2)^{(L)}$ gauging is based on a manifest symmetry of the Cartan integrable system. 
Going to the Vasiliev gauge, the master fields obey the following similarity transformed $s p(2)^{(L)}$ invariance conditions:

$$
\left[\Phi, K_{i j}^{(L)}\right]_{\star}=0, \quad D K_{i j}^{(L)} \equiv d K_{i j}^{(L)}+\left[W, K_{i j}^{(L)}\right]_{\star}=0,
$$

where

$$
K_{i j}^{(L)}:=L^{-1} \star K_{i j}^{(Y)} \star L=H^{-1} \star K_{i j}^{(Y)} \star H,
$$

are field dependent generators such that

$$
\left(K_{i j}^{(L)}\right)^{(0)}=K_{i j}^{(Y)} .
$$

Comparison of old and new Type A models. In the Vasiliev gauge, and prior to factoring out the $s p(2)$ ideal, both models have perturbatively defined solution spaces obeying the same differential equations, gauge conditions, viz.

$$
W_{a i}=0, \quad Z^{i}\left[W_{i}\right]_{\mathrm{n}}=0,
$$

and $s p(2)$ invariance conditions, viz.

$$
D_{i} K_{j k}=0, \quad\left[K_{i j}, \Phi\right]_{\pi}=0, \quad\left[K_{i j}, K_{k l}\right]_{\star}=4 i \epsilon_{j k} K_{i l},
$$

with $s p(2)$ generators subject to the same functional initial condition, viz.

$$
\left.K_{i j}\right|_{\Phi=0}=K_{i j}^{(Y)} .
$$

This suggests that the two models are perturbatively equivalent, modulo redefinitions of zero-form initial data and modifications of the Vasiliev gauge condition away from the asymptotic region. This can be examined by comparing the first sub-leading corrections $K_{i j}^{(L)}$ and $K_{i j}^{\text {(diag) }}$, which we leave for a separate work.

\subsubsection{Factoring out the $\operatorname{sp}(2)$ ideal}

Thus, so far we have obtained perturbatively defined configurations (3.9) of both models, which obey the same differential constraints with $s p(2)^{(Y)}$ invariant zero-form initial data ensuring the full $s p(2)$ invariance condition. In order to go fully on-shell it remains to factor out the $s p(2)$ orbits from these solution spaces. As we shall see next, this operation combines naturally with the problem of constructing higher spin gauge invariant functionals.

Quasi-projector. Letting $\mathcal{E}_{0}$ denote the space of $s p(2)$ invariant horizontal forms on $\mathcal{C}$, its (two-sided) ideal $\mathcal{I}$ generated by $K_{i j}$ can be factored out by using the modified trace operation

$$
\operatorname{Tr}_{\Delta} f:=\int_{\mathcal{Y} \times \mathcal{Z}}[V \star \Delta \star f]_{\mathrm{b}}, \quad[f] \in \mathcal{E}_{0} / \mathcal{I}
$$

where $[\cdot]_{\mathrm{b}}$ is the Wigner map in a given operator order $\mathrm{b} ; V$ is the volume form on $\mathcal{Y}$; and $\Delta$ is a horizontal zero-form obeying

$$
K_{i j} \star \Delta=0=\Delta \star K_{i j}
$$


the covariant constancy condition

$$
D_{M} \Delta=0, \quad[\Delta, \Phi]_{\pi}=0
$$

and the quasi-projector property, namely that

$$
\Delta \star \mathcal{E}_{0} \cong \mathcal{E}_{0} / \mathcal{I}
$$

as a vector space, while $\Delta \star \Delta \star \mathcal{E}_{0}$ need not exist. Provided that the trace operation is well-defined it is independent of the details going into $[\cdot]_{\mathrm{b}}$; in particular, it can be performed in Weyl as well as normal order.

It follows that

$$
D_{M}(\Delta \star \Phi \star \kappa)=0, \quad D_{M}\left(\Delta \star S_{A i}\right)=0,
$$

of which the first equation indeed contains the correct linearized mass-shell conditions for generalized Weyl tensors (including the dynamical scalar field).

In the new model, we have [41]

$$
\Delta^{(Y)}=F\left(K^{i j(Y)} K_{i j}^{(Y)}\right), \quad F(z)=z^{-\nu / 2} I_{\nu}(\sqrt{2 z}), \quad \nu=\frac{D}{2}-1,
$$

in the integrable gauge, where $I_{\nu}(x)$ is the modified Bessel function such that $F$ is real analytic and non-vanishing at the origin, and

$$
\Delta^{(L)}=L^{-1} \star \Delta^{(Y)} \star L,
$$

in the Vasiliev gauge.

In the old model, $\Delta^{(\text {diag })}$ can instead be found by solving $K_{i j}^{(\text {diag })} \star \Delta^{(\text {diag })}=0$ perturbatively [41].

Zero-form charges. The simplest observables are the zero-form charges $[29,31]$

$$
\mathcal{O}_{C}:=\operatorname{Tr}_{\Delta} J \star J^{\prime} \star \mathcal{W}_{C},
$$

where $J^{\prime}$ is a volume form on the $2 D$-dimensional subspace of $\mathcal{Z}$ coordinatized by $Z^{a i}$, and $\mathcal{W}_{C}$ are twisted (open) Wilson lines in $\mathcal{Z}$ from $Z=0$ to $Z=M$ which can be straightened out into star products of vertex-like operators [34, 42], viz.

$$
\mathcal{W}_{C}=f_{C}(\Phi \star \kappa) \star \mathcal{V}_{\Lambda}, \quad \mathcal{V}_{\Lambda}:=\exp _{\star}\left(i M^{A i} S_{A i}\right),
$$

where $f_{C}$ is given by an expansion in terms of $(\Phi \star \kappa)^{\star n}(n=0,1,2, \ldots)$ depending on the shape of $C$. The zero-form charges are de Rham closed on $\mathcal{X}$, and hence higher spin gauge invariant on-shell by virtue of

$$
\partial_{M} \mathcal{O}=\int_{\mathcal{Y} \times \mathcal{Z}}\left[\partial_{M}\left(\Delta \star V \star J \star J^{\prime} \star \mathcal{W}_{C}\right)\right]_{\mathrm{b}}=\int_{\mathcal{Y} \times \mathcal{Z}}\left[D_{M}\left(\Delta \star V \star J \star J^{\prime} \star \mathcal{W}_{C}\right)\right]_{\mathrm{b}}=0,
$$

using $D_{M} \mathcal{W}_{C}=0$. 
$\boldsymbol{p}$-form charges. More general invariants [5, 31], that can be evaluated on non-trivial elements $[\Sigma]$ in the singular homology of $\mathcal{X}$, can be constructed by choosing a structure group $G$ with connection $\Omega_{M}$ and splitting

$$
W_{M}=\Omega_{M}+E_{M},
$$

where $E_{M}$ is a soldering one-form, that is, a generalized frame field, whose gauge parameters belong to sections that can be converted to globally defined vector fields on $\mathcal{X}$ (modulo a $G$ gauge transformation with composite parameter). This facilitates the definition of $G$ invariant tensors on $\mathcal{X}$, which induce top forms on representatives $\Sigma^{\prime} \in[\Sigma]$ whose integrals over $\Sigma^{\prime}$ define generalized volumes whose extrema (as one varies $\Sigma^{\prime}$ ) are diffeomorphism invariants, and hence higher spin gauge invariant by the soldering mechanism. These geometries also support closed abelian even forms

$$
H_{[2 p]}=\operatorname{Tr}_{\Delta}(E \star E)^{\star p},
$$

on $\mathcal{X}$, whose charges $\oint_{\Sigma} H_{[2 p]}$ are higher spin gauge invariant.

As first suggested in [14], the zero-form charges have perturbative expansions over asymptotically anti-de Sitter solutions in terms of boundary correlation function, as has been verified and developed further in the context of four-dimensional spinor oscillator models [30, 32, 33], where it has also been proposed [39] that they can be interpreted as extensive charges for families of localizable black-hole like solutions. Thus, zero-form charges together with other invariants could serve as tools for establishing the perturbative equivalence between the old and new Type A models. ${ }^{6}$

\section{Frobenius-Chern-Simons extension}

In this section, we first couple the new Type A model to a dynamical two-form leading to a model formulated in terms of a superconnection, suitable for making contact with topological open strings. We then introduce $s p(2)$ ghosts to the oscillator algebra and related additional auxiliary fields so as to incorporate the $s p(2)$ gauging into the extended Cartan gauge structure. The resulting equations of motion are variational with a FrobeniusChern-Simons action.

\subsection{Dynamical two-form}

It is natural to seek an extension of the Vasiliev system such that the rigid two-form $J$ arises as a vacuum expectation value of a dynamical two-form.

To this end, we first introduce a pair $(A, \widetilde{A})$ of one-forms in $\mathcal{E}$, with curvatures

$$
F:=d A+A \star A, \quad \widetilde{F}:=d \widetilde{A}+\widetilde{A} \star \widetilde{A},
$$

and a pair $(\Phi, \tilde{\Phi})$ of forms in $\mathcal{E}$ of degrees $(0,2)$ in opposite twisted bi-fundamental representations, as dictated by the covariant derivatives

$$
D \Phi:=d \Phi+A \star \Phi-\Phi \star \pi(\widetilde{A}), \quad \widetilde{D} \widetilde{\Phi}:=d \widetilde{\Phi}+\pi(\widetilde{A}) \star \widetilde{\Phi}-\widetilde{\Phi} \star A,
$$

\footnotetext{
${ }^{6}$ They could also be useful in establishing the equivalence between the vector and spinor oscillator formulations of the Type A model in four dimensions.
} 
such that $\Phi \star \widetilde{\Phi}$ and $\pi(\widetilde{\Phi} \star \Phi)$ can be used to source $F$ and $\widetilde{F}$, respectively. We also introduce two separate sets of $s p(2)$ generators $\left(K_{i j}, \widetilde{K}_{i j}\right)$ in form degree zero, viz.

$$
\left[K_{i j}, K_{k l}\right]_{\star}=4 i \epsilon_{(i \mid(k} K_{l) \mid j)}, \quad\left[\widetilde{K}_{i j}, \widetilde{K}_{k l}\right]_{\star}=4 i \epsilon_{(i \mid(k} \widetilde{K}_{l) \mid j)},
$$

with covariant derivatives

$$
D K_{i j}:=d K_{i j}+A \star K_{i j}-K_{i j} \star A, \quad \widetilde{D} \widetilde{K}_{i j}:=d \widetilde{K}_{i j}+\widetilde{A} \star \widetilde{K}_{i j}-\widetilde{K}_{i j} \star \widetilde{A} .
$$

The Cartan integrable equations of motion of the extended system read as follows:

$$
\begin{aligned}
F+\Phi \star \widetilde{\Phi} & =0, & & 0=\widetilde{F}+\pi(\widetilde{\Phi} \star \Phi), \\
D \Phi & =0, & & 0=\widetilde{D} \widetilde{\Phi} \\
D K_{i j} & =0, & & 0=\widetilde{D} \widetilde{K}_{i j}, \\
K_{i j} \star \Phi-\Phi \star \pi\left(\widetilde{K}_{i j}\right) & =0, & & =\pi\left(\widetilde{K}_{i j}\right) \star \widetilde{\Phi}-\widetilde{\Phi} \star K_{i j} .
\end{aligned}
$$

The field configurations are considered to be equivalent if they belong to the same orbit generated by the shift transformations

$$
\begin{aligned}
\delta_{\mathcal{I}} A & =K_{i j} \star \alpha^{i j}, & \delta_{\mathcal{I}} \widetilde{A} & =\widetilde{K}_{i j} \star \tilde{\alpha}^{i j}, \\
\delta_{\mathcal{I}} \Phi & =K_{i j} \star \varphi^{i j}, & \delta_{\mathcal{I}} \widetilde{\Phi} & =\pi\left(\widetilde{K}_{i j}\right) \star \widetilde{\varphi}^{i j} \\
\delta_{\mathcal{I}} K_{i j} & =0, & \delta_{\mathcal{I}} \widetilde{K}_{i j} & =0,
\end{aligned}
$$

where the parameters $\left(\alpha^{i j}, \tilde{\alpha}^{i j} ; \varphi^{i j}, \tilde{\varphi}^{i j}\right)$ are forms in degrees $(1,1 ; 0,2)$ obeying

$$
\begin{array}{rlrl}
{\left[K_{i j}, \alpha_{k l}\right]_{\star}=4 i \epsilon_{(i \mid(k} \alpha_{l) \mid j)},} & {\left[\widetilde{K}_{i j}, \tilde{\alpha}_{k l}\right]_{\star}} & =4 i \epsilon_{(i \mid(k} \tilde{\alpha}_{l) \mid j)}, \\
K_{i j} \star \varphi_{k l}-\varphi_{k l} \star \pi\left(\widetilde{K}_{i j}\right) & =4 i \epsilon_{(i \mid(k} \varphi_{l) \mid j)}, & \pi\left(\widetilde{K}_{i j}\right) \star \tilde{\varphi}_{k l}-\tilde{\varphi}_{k l} \star K_{i j} & =4 i \epsilon_{(i \mid(k} \tilde{\varphi}_{l) \mid j)} .
\end{array}
$$

Finally, the reality conditions read

$$
A^{\dagger}=-\widetilde{A}, \quad \Phi^{\dagger}=\pi(\Phi), \quad \widetilde{\Phi}^{\dagger}=-\pi(\widetilde{\Phi}), \quad\left(K_{i j}\right)^{\dagger}=\widetilde{K}_{i j}
$$

Outer Klein operator. The equations can be re-written by introducing an outer Klein operator $k$ that obeys $k^{2}=1$ along with

$$
\left[k, Y_{i}^{a}\right]=0, \quad\left\{k, Y_{i}\right\}=0, \quad\left[k, Z_{i}^{a}\right]=0, \quad\left\{k, Z_{i}\right\}=0, \quad d k=k d,
$$

and defining

$$
B=\Phi k, \quad \widetilde{B}=k \widetilde{\Phi},
$$

after which the equations of motion read

$$
\begin{aligned}
F+B \star \widetilde{B} & =0, & & 0=\widetilde{F}+\widetilde{B} \star B, \\
D B & =0, & 0 & =\widetilde{D} \widetilde{B}, \\
D K_{i j} & =0, & 0 & =\widetilde{D} \widetilde{K}_{i j}, \\
K_{i j} \star B-B \star \widetilde{K}_{i j} & =0, & 0 & =\widetilde{K}_{i j} \star \widetilde{B}-\widetilde{B} \star K_{i j},
\end{aligned}
$$


where now

$$
D B:=d B+A \star B-B \star \widetilde{A}, \quad \widetilde{D} \widetilde{B}:=d \widetilde{B}+\widetilde{A} \star \widetilde{B}-\widetilde{B} \star A .
$$

The modified shift transformations read

$$
\delta_{\mathcal{I}} B=K_{i j} \star \beta^{i j}, \quad \delta_{\mathcal{I}} \widetilde{B}=\widetilde{K}_{i j} \star \widetilde{\beta}^{i j}
$$

where $\beta_{i j}=\varphi_{i j} k, \tilde{\beta}_{i j}=k \tilde{\varphi}_{i j}$ and

$$
K_{i j} \star \beta_{k l}-\beta_{k l} \star \widetilde{K}_{i j}=4 i \epsilon_{(i \mid(k} \beta_{l) \mid j)}, \quad \widetilde{K}_{i j} \star \tilde{\beta}_{k l}-\tilde{\beta}_{k l} \star K_{i j}=4 i \epsilon_{(i \mid(k} \tilde{\beta}_{l) \mid j)} .
$$

The reality conditions are

$$
A^{\dagger}=-\widetilde{A}, \quad B^{\dagger}=B, \quad \widetilde{B}^{\dagger}=-\widetilde{B} .
$$

Finally, the system can be extended further by allowing $(A, \widetilde{A} ; B, \widetilde{B})$ to have a general dependence on $k$, after which the previous system is recovered by defining $\Phi:=B k$ and $\widetilde{\Phi}:=k \widetilde{B}$ and taking $(A, \widetilde{A}, \Phi, \widetilde{\Phi})$ to be $k$-independent.

Obstruction of $\operatorname{sp(2)}$ gauging from dynamical two-form. Prior to eliminating $k$, the one-form $S:=d Z^{A i} S_{A i}$ with $S_{A i}:=Z_{A i}-2 i A_{A i}$ obeys

$$
\begin{aligned}
{\left[S_{a i}, S_{b j}\right]_{\star} } & =2 \imath_{b j} \imath_{a i}(S \star S), \\
\pi_{k}\left(S_{A i}\right) \star S_{j}-S_{j} \star S_{A i} & =2 \imath_{j} \imath_{A i}(S \star S),
\end{aligned}
$$

where

$$
S \star S=i d Z^{A i} d Z_{A i}+4 B \star \widetilde{B},
$$

and the inner derivatives $\imath_{A i} \equiv \imath_{\partial_{A i}}$ act from the left, using the rule $\left[k, \imath_{a i}\right]=0$ and $\left\{k, \imath_{i}\right\}=0$. In deriving (4.25) we have used $\left\{d Z^{A i} Z_{A i}, A\right\}=-2 i d Z^{A i} \partial_{A i} A$ and $F=-B \star \widetilde{B}$. Thus, after eliminating $k$, we have ${ }^{7}$

$$
\left[S_{A i}, S_{B j}\right]_{\star}=2 \imath_{B j} \imath_{A i}(S \star S), \quad S \star S=i d Z^{A i} d Z_{A i}+4 \Phi \star \widetilde{\Phi},
$$

that is, the introduction of the dynamical two-form obstructs the Wigner deformed oscillator algebra, and hence the diagonal $s p(2)$ algebra on-shell.

Instead, one may set

$$
K_{i j}=\widetilde{K}_{i j}=K_{i j}^{(Y)},
$$

for general two-form backgrounds. For this choice, and assuming that $\mathcal{Z}$ contains an $S^{2}$ on which $\widetilde{B}$ can be wrapped as to produce $J$ as a vacuum expectation value, the consistent truncation $^{8}$

$$
\widetilde{\Phi}=J, \quad \widetilde{A}=A=W,
$$

yields the new Type A model in its integrable gauge. There remains, however, an implicit obstruction of the $s p(2)$ gauging, as the map to the Vasiliev gauge refers to the gauge function $L$ which requires $W$ to be flat on $\mathcal{X}$.

\footnotetext{
${ }^{7}$ The one-form $\widetilde{S}:=d Z^{A i} \widetilde{S}_{A i}$ with $\widetilde{S}_{A i}:=Z_{A i}-2 i \widetilde{A}_{A i}$ obeys similar constraints, and there is no constraint on mutual star products between $S_{A i}$ and $\widetilde{S}_{A i}$ master fields.

${ }^{8}$ The non-trivial two-cycle in $\mathcal{Z}$ implies that the dynamical two-form contains additional degrees of freedom; for a related feature in the case of the spinorial formulation of higher spin gravity in four spacetime dimensions, see [10, 27].
} 


\subsection{Superconnection}

As topological two-dimensional sigma models provide a first-quantized description of noncommutative differential form algebras on Poisson manifolds [15-17, 43-47], it is desirable to cast the equations of motion into a format more akin to that of a topological open string field theory, namely a flatness condition on a graded odd superconnection in the direct product of $\mathcal{E}$ and a suitably graded internal Frobenius algebra $\mathcal{F}$ [13].

Duality extension. We first extend the field content by taking $(A, \widetilde{A}, B, \widetilde{B})$ to be horizontal forms of degrees $(1,1,0,2) \bmod 2$, respectively. We can reduce the $k$-dependence by defining $\Phi=B k$ and $\widetilde{\Phi}=k \widetilde{B}$ and taking $(A, \widetilde{A}, \Phi, \widetilde{\Phi})$ to be $k$-independent forms of degrees $(1,1,0,2)$ mod 2 , respectively, which yields the duality extension of the system with twisted bi-fundamental zero-form and two-form.

Frobenius algebra. We take $\mathcal{F} \equiv \operatorname{Mat}_{2}(\mathbb{C})$ to be spanned by $(I, J=1,2)[10,27]$

$$
\boldsymbol{e}_{I J}=\left[\begin{array}{cc}
\boldsymbol{e} & \boldsymbol{f} \\
\widetilde{\boldsymbol{f}} & \widetilde{\boldsymbol{e}}
\end{array}\right], \quad e_{I J} e_{K L}=\delta_{J K} e_{I L},
$$

introduce the 3 -grading

$$
\operatorname{deg}_{\mathcal{F}}(\tilde{\boldsymbol{f}}, \boldsymbol{e}, \widetilde{\boldsymbol{e}}, \boldsymbol{f})=(-1,0,0,1,),
$$

and use Koszul signs governed by the total degree

$$
\operatorname{deg}:=\operatorname{deg}_{\mathcal{E}}+\operatorname{deg}_{\mathcal{F}} .
$$

We can then define the superconnection, nilpotent differential and supercovariant derivative, respectively, by

$$
\mathbf{X}:=\boldsymbol{e} A+\widetilde{\boldsymbol{e}} \widetilde{A}+\boldsymbol{f} B+\widetilde{\boldsymbol{f}} \widetilde{B}, \quad \mathbf{q}:=(\boldsymbol{e}+\widetilde{\boldsymbol{e}}) d, \quad \boldsymbol{D}:=\mathbf{q}+\operatorname{ad}_{\mathbf{X}}^{\star},
$$

with degrees

$$
\operatorname{deg}(\mathbf{X}) \in\{1,3, \ldots\}, \quad \operatorname{deg}(\mathbf{q})=\operatorname{deg}(\boldsymbol{D})=1 .
$$

We also define $s p(2)$ generators by

$$
\mathbf{K}_{i j}:=\boldsymbol{e} K_{i j}+\widetilde{\boldsymbol{e}} \widetilde{K}_{i j}, \quad \text { with } \operatorname{deg}\left(\mathbf{K}_{i j}\right)=0 .
$$

In terms of these requisites, the equations of motion and $s p(2)$ gauge conditions take the desired form,

$$
\begin{aligned}
\mathbf{q} \mathbf{X}+\mathbf{X} \star \mathbf{X} & =0, \\
\mathbf{D} \mathbf{K}_{i j} & =0, \quad\left[\mathbf{K}_{i j}, \mathbf{K}_{k l}\right]_{\star}=4 i \epsilon_{(i \mid(k} \mathbf{K}_{l) \mid j)},
\end{aligned}
$$

whose local symmetries are thus given by the Cartan gauge transformations

$$
\delta_{\boldsymbol{\epsilon}} \mathbf{X}=\boldsymbol{D} \boldsymbol{\epsilon}, \quad \delta_{\boldsymbol{\epsilon}} \mathbf{K}_{i j}=\left[\mathbf{K}_{i j}, \boldsymbol{\epsilon}\right]_{\star},
$$

and the ideal shift symmetries

$$
\delta_{\mathcal{I}} \mathbf{X}=\mathbf{K}_{i j} \star \boldsymbol{\xi}^{i j}, \quad \delta_{\mathcal{I}} \mathbf{K}_{i j}=0,
$$

where

$$
\boldsymbol{\xi}_{i j}=\alpha_{i j} \boldsymbol{e}+\tilde{\alpha}_{i j} \widetilde{\boldsymbol{e}}+\beta_{i j} \boldsymbol{f}+\tilde{\beta}_{i j} \tilde{\boldsymbol{f}}, \quad \operatorname{deg}\left(\boldsymbol{\xi}_{i j}\right) \in\{1,3, \ldots\},
$$

obeying $\left[\mathbf{K}_{i j}, \boldsymbol{\xi}_{k l}\right]=4 i \epsilon_{(i \mid(k} \boldsymbol{\xi}_{l) \mid j}$. 


\subsection{Action with dynamical two-form}

In what follows, we use first-quantized $s p(2)$ ghosts to extend the equations of motion (4.37) and (4.38) as to reach a set that is variational under the assumption that the $s p(2)$ generators $\mathbf{K}_{i j}$ are given by the undeformed $\mathbf{K}_{i j}^{(Y)}$ off-shell.

First-quantized ghosts. We thus extend the fiber algebra by the Clifford algebra $\mathcal{G}$ generated by $\left(\widehat{B}_{i j}, \widehat{C}^{i j}\right)$ obeying ${ }^{9}$

$$
\left[\widehat{B}^{i j}, \widehat{C}_{k l}\right]_{\star}=2 \delta_{(k}^{i} \delta_{l)}^{j}, \quad \operatorname{deg}_{\mathcal{G}}\left(\widehat{B}^{i j}, \widehat{C}_{i j}\right)=(-1,1),
$$

and let the Koszul signs of $\mathcal{E} \otimes \mathcal{F} \otimes \mathcal{G}$ be governed by the total degree

$$
|\cdot|=\operatorname{deg}_{\mathcal{E}}+\operatorname{deg}_{\mathcal{F}}+\operatorname{deg}_{\mathcal{G}} .
$$

The BRST operator

$$
\boldsymbol{Q}^{(Y)}:=\widehat{C}^{i j} \mathbf{K}_{i j}^{(Y)}-2 i(\boldsymbol{e}+\widetilde{\boldsymbol{e}}) \widehat{B}_{i}{ }^{j} \widehat{C}_{j}{ }^{k} \widehat{C}_{k}{ }^{i},
$$

obeys

$$
\boldsymbol{Q}^{(Y)} \star \boldsymbol{Q}^{(Y)}=0, \quad \mathbf{q} \boldsymbol{Q}^{(Y)}=0,
$$

and hence the differential

$$
\mathbf{q}^{(Y)}:=\mathbf{q}+\operatorname{ad}_{\boldsymbol{Q}^{(Y)}}^{\star},
$$

is nilpotent, viz.

$$
\left(\mathbf{q}^{(Y)}\right)^{2}=0 .
$$

Extended master fields and boundary conditions. We introduce two horizontal forms $(\widehat{\mathbf{X}}, \widehat{\mathbf{P}})$ of total degrees

$$
\begin{aligned}
& |\widehat{\mathbf{X}}| \in 2 \mathbb{Z}+1, \\
& |\widehat{\mathbf{P}}| \in \begin{cases}2 \mathbb{Z}+1 \text { if } \operatorname{dim}(\mathcal{X}) \text { odd } \\
2 \mathbb{Z} & \text { if } \operatorname{dim}(\mathcal{X}) \text { even }\end{cases}
\end{aligned}
$$

We furthermore assume that

$$
\operatorname{dim}(\mathcal{X})=D+1,
$$

and impose boundary conditions on the elements of $\mathcal{E}$ such that $[10,27]$

$-\partial \mathcal{Z}=\emptyset$

- $\partial \mathcal{X}$, which is a $D$-dimensional compact manifold, has marked submanifolds where sections blow up with tubular neighborhoods identifiable as asymptotically anti-de Sitter regions;

- $\mathcal{A}$ is a non-polynomial completion of the extended Weyl algebra generated by polynomials in $Y, \kappa_{y}$ and $k$, with trace operation $\operatorname{Tr}_{\mathcal{A}}$.

\footnotetext{
${ }^{9}$ We use conventions in which all star brackets are graded, and we let hats denote quantities with generic dependence on ghost variables.
} 
We furthermore impose

$$
\left.\widehat{\mathbf{P}}\right|_{\partial(\mathcal{X} \times \mathcal{Z})}=0
$$

as to ensure a smooth BV-BRST differential [26, 48], and restrict

$$
\begin{aligned}
\operatorname{deg}(\widehat{\mathbf{X}}) & \in\{1,2,3, \ldots, 3(D+1)-1\}, \\
\operatorname{deg}(\widehat{\mathbf{P}}) & \in\{0,1,2, \ldots, 3(D+1)-2\} .
\end{aligned}
$$

Action. Applying the variational principle to the functional

$$
\begin{aligned}
\operatorname{dim}(\mathcal{X}) \text { odd }: & S=\int_{\mathcal{X} \times \mathcal{Z}} \operatorname{Tr}_{\mathcal{A}} \operatorname{Tr}_{\mathcal{F}} \operatorname{Tr}_{\mathcal{G}}\left(\widehat{\mathbf{P}} \star\left(\mathbf{q}^{(Y)} \widehat{\mathbf{X}}+\widehat{\mathbf{X}} \star \widehat{\mathbf{X}}\right)+\frac{1}{3} \widehat{\mathbf{P}} \star \widehat{\mathbf{P}} \star \widehat{\mathbf{P}}\right), \\
\operatorname{dim}(\mathcal{X}) \text { even }: & S=\int_{\mathcal{X} \times \mathcal{Z}} \operatorname{Tr}_{\mathcal{A}} \operatorname{Tr}_{\mathcal{F}} \operatorname{Tr}_{\mathcal{G}}\left(\widehat{\mathbf{P}} \star\left(\mathbf{q}^{(Y)} \widehat{\mathbf{X}}+\widehat{\mathbf{X}} \star \widehat{\mathbf{X}}\right)+\frac{1}{2} \widehat{\mathbf{P}} \star \widehat{\mathbf{P}}\right),
\end{aligned}
$$

where $\operatorname{Tr}_{\mathcal{F}}$ and $\operatorname{Tr}_{\mathcal{G}}$, respectively, denote the (cyclic) trace operations on $\mathcal{F}$ and $\mathcal{G}$ generated by the ghosts, yields the Cartan integrable equations of motion

$$
\mathbf{q}^{(Y)} \widehat{\mathbf{X}}+\widehat{\mathbf{X}} \star \widehat{\mathbf{X}}+\widehat{\mathbf{P}} \star \widehat{\mathbf{P}} \approx 0, \quad \mathbf{q}^{(Y)} \widehat{\mathbf{P}}+[\widehat{\mathbf{X}}, \widehat{\mathbf{P}}]_{\star} \approx 0
$$

and the Cartan gauge transformations leave the action invariant provided that the gauge parameter of $\widehat{\mathbf{P}}$ vanishes at $\partial \mathcal{X} \times \mathcal{Z}$.

Ghost number truncation and $s p(2)$ auxiliary fields. The boundary equations of motion

$$
\mathbf{q}^{(Y)} \widehat{\mathbf{X}}+\widehat{\mathbf{X}} \star \widehat{\mathbf{X}} \approx 0, \quad \text { at } \partial \mathcal{X} \times \mathcal{Z}
$$

can be truncated by taking

$$
\widehat{\mathbf{X}}=\sum_{n=1}^{4} \widehat{\mathbf{X}}_{n}, \quad\left(\operatorname{deg}, \operatorname{deg}_{\mathcal{G}}\right)\left(\widehat{\mathbf{X}}_{n}\right)=(n, 1-n),
$$

where thus $\widehat{\mathbf{X}}_{m} \star \widehat{\mathbf{X}}_{n} \equiv 0$ if $m+n \geqslant 6$, which yields the quasi-free differential algebra with algebraic constraint

$$
\left[\boldsymbol{Q}^{(Y)}, \widehat{\mathbf{X}}_{1}\right]_{\star}=0
$$

and differential constraints ${ }^{10}$

$$
\begin{aligned}
& \mathbf{q} \widehat{\mathbf{X}}_{1}+\widehat{\mathbf{X}}_{1} \star \widehat{\mathbf{X}}_{1}+\left[\boldsymbol{Q}^{(Y)}, \widehat{\mathbf{X}}_{2}\right]_{\star}=0, \\
& \widehat{\mathbf{D}} \widehat{\mathbf{X}}_{n}+\left[\boldsymbol{Q}^{(Y)}, \widehat{\mathbf{X}}_{n+1}\right]_{\star}+\sum_{\substack{n_{1}+n_{2}=n+1 \\
n_{1,2} \geqslant 2}} \widehat{\mathbf{X}}_{n_{1}} \star \widehat{\mathbf{X}}_{n_{2}}=0, \quad n=2,3,4,
\end{aligned}
$$

${ }^{10}$ The Cartan gauge transformations

$$
\delta \widehat{\mathbf{X}}_{n}=\widehat{\mathbf{D}} \widehat{\boldsymbol{\epsilon}}_{n-1}+\left[\boldsymbol{Q}^{(Y)}, \widehat{\boldsymbol{\epsilon}}_{n}\right]+\sum_{\substack{n_{1}+n_{2}=n \\ n_{1}>1}}\left[\widehat{\mathbf{X}}_{n_{1}}, \widehat{\boldsymbol{\epsilon}}_{n_{2}}\right]_{\star}, \quad n=1,2, \ldots, 4
$$

where $\operatorname{deg}\left(\widehat{\boldsymbol{\epsilon}}_{n}\right)=n$. 
where

$$
\widehat{\mathbf{D}}:=\mathbf{q}+\operatorname{ad}_{\widehat{\mathbf{X}}_{1}}^{\star},
$$

which provides an extension of the system in eqs. (4.37) and (4.38) via the identifications

$$
\mathbf{K}_{i j}=\mathbf{K}_{i j}^{(Y)}, \quad \mathbf{X}=\left.\widehat{\mathbf{X}}_{1}\right|_{\widehat{B}=0=\widehat{C}} .
$$

We shall notice that all the field equations presented so far where meant to hold up to terms proportional to ideal elements of the form $\mathcal{O}^{i j} \star K_{i j}^{(Y)}$. By extending the field content via the first-quantized ghosts, we have both shift symmetries and ideal source terms automatically incorporated in the Cartan integrable system (4.59)-(4.61) via the $\boldsymbol{Q}^{(Y)}$ exact commutators. The remaining component fields in the ghost expansions of $\widehat{\mathbf{X}}_{n}, n=$ $1, \ldots, 4$, provide a complete set of $s p(2)$ auxiliary fields ensuring Cartan integrability, that include now the $s p(2)$ shifts as gauge transformations. The system admits the consistent truncation

$$
\widehat{\mathbf{X}}_{1}=\mathbf{X}, \quad \widehat{\mathbf{X}}_{n}=0, \quad n=2,3,4,
$$

which yields

$$
\boldsymbol{Q}^{(Y)} \star \boldsymbol{Q}^{(Y)}=0, \quad\left[\boldsymbol{Q}^{(Y)}, \mathbf{X}\right]_{\star}=0, \quad \mathbf{q} \mathbf{X}+\mathbf{X} \star \mathbf{X}=0,
$$

with gauge transformations $\delta \mathbf{X}=\boldsymbol{D} \epsilon_{0}$, where the gauge parameter is constrained to $\left[\boldsymbol{Q}^{(Y)}, \mathbf{X}\right]_{\star}=0$.

Treating $\mathbf{X}$ as a zeroth order background, switching on $\boldsymbol{h}_{i j}:=\left.\frac{\partial}{\partial \widehat{B}^{i j}} \widehat{\mathbf{X}}_{2}\right|_{\widehat{B}=0=\widehat{C}}$, treated as a first-order perturbation, induces perturbative corrections $\widehat{\mathbf{X}}_{n}^{(p)}, p=1, \ldots$ At first order, we thus have the perturbation $\widehat{\mathbf{X}}_{2}^{(1)}=\frac{1}{2} \widehat{B}_{i j} \boldsymbol{h}^{i j}$ inducing $\widehat{\mathbf{X}}_{1}^{(1)}=\mathbf{X}^{(1)}$, a deformation of $\mathbf{X}$, but not any onset of any further $s p(2)$ auxiliary fields. At second order, however, $\widehat{\mathbf{X}}_{3}^{(2)}$ contains new auxiliary fields, inducing $\widehat{\mathbf{X}}_{2}^{(2)}$ and $\widehat{\mathbf{X}}_{1}^{(2)}$, containing further deformations $\boldsymbol{h}_{i j}^{(2)}$ and $\mathbf{X}^{(2)}$ as well as new auxiliary fields. In general, the full perturbative expansion will activate the full master fields (subject to the ghost number truncation).

Classical observables and first-quantized physical state quasi-projector. As for classical observables, we use the fact that starting from a Lie algebra with generators $T_{\alpha}$ and ghosts $\left(B_{\alpha}, C^{\beta}\right)$

$$
\left[T_{\alpha}, T_{\beta}\right]_{\star}=i f_{\alpha \beta}^{\gamma} T_{\gamma}, \quad\left[B_{\alpha}, C^{\beta}\right]_{\star}=2 \delta_{\alpha}^{\beta},
$$

where $f_{\alpha \beta}{ }^{\beta}=0$, the BRST operator

$$
Q=C^{\alpha} \star\left(T_{\alpha}+\frac{1}{2} T_{\alpha}^{(g h)}\right), \quad T_{\alpha}^{(g h)}:=\frac{i}{2} f_{\alpha \beta}{ }^{\gamma} B_{\gamma} C^{\beta},
$$

using Weyl ordered symbols. Thus, given an operator $\mathcal{O}$ in the BRST cohomology, that is,

$$
[Q, \mathcal{O}]_{\star}=0, \quad \delta_{\mathcal{X}} \mathcal{O}=[Q, \mathcal{X}]_{\star},
$$

we can define its expectation value by

$$
\langle\mathcal{O}\rangle=\operatorname{Tr}\left(\Delta^{(\text {tot })} \star \mathcal{O}\right), \quad Q \star \Delta^{(\text {tot })}=0=\Delta^{(\text {tot })} \star Q,
$$

where the trace is over the total space. 
In the case of $s p(2)^{(Y)}$, the quasi-projector $\Delta^{(\text {tot })}$ can be factorized as

$$
\Delta^{(\text {tot })}=\Delta^{(Y)} \star \Delta^{(\mathrm{gh})}, \quad K_{i j}^{(Y)} \star \Delta^{(Y)}=0, \quad K_{i j}^{(g h)} \star \Delta^{(\mathrm{gh})}=0,
$$

where

$$
\Delta^{(Y)}=z^{-\nu / 2} I_{\nu}(\sqrt{2 z}), \quad z=K^{(Y) i j} K_{i j}^{(Y)}, \quad \nu=\frac{D-2}{2},
$$

as can be seen using $K_{i j}^{(Y)} \star f(z)=K_{i j}^{(Y)}\left(1-D \partial_{z}-2 z \partial_{z}^{2}\right) f$ and

$$
\Delta^{(\mathrm{gh})}=\mu_{1}\left(1-\frac{1}{8} z\right)+\mu_{2}\left(w+\frac{1}{6} w^{3}\right), \quad z:=K^{(g h) i j} K_{i j}^{(g h)}, \quad w=B^{i j} C_{i j},
$$

where $\mu_{1}, \mu_{2} \in \mathbb{R}$, as can be seen using the fact that there are four singlets in ghost number zero, given by $1, w, z, w^{3}$, and that

$$
K_{i j}^{(g h)} \star z=8 K_{i j}^{(g h)}, \quad K_{i j}^{(g h)} \star w=K_{i j}^{(g h)} w, \quad K_{i j}^{(g h)} \star w^{3}=-6 K_{i j}^{(g h)} w,
$$

where $K_{i j}^{(g h)}=2 i B_{(i}{ }^{k} C_{j) k}$. Taking $\mathcal{O}=\mathcal{O}^{(Y)}$ to be independent of the ghosts, we have

$$
\left[K_{i j}^{(Y)}, \mathcal{O}^{(Y)}\right]_{\star}=0, \quad \delta_{\mathcal{X}} \mathcal{O}^{(Y)}=K_{i j}^{(Y)} \star \xi^{(Y) i j},
$$

where $\mathcal{X}=\frac{1}{2} B_{i j} \star \xi^{(Y) i j}=\frac{1}{2} B_{i j} \xi^{(Y) i j}$ with $\xi^{(Y) i j}$ being independent of the ghosts, and

$$
\langle\mathcal{O}\rangle=\mu_{2} \operatorname{Tr}_{\mathcal{A}} \Delta^{(Y)} \star \mathcal{O}^{(Y)},
$$

using $\operatorname{Tr}=\operatorname{Tr}_{\mathcal{A}} \operatorname{Tr}_{\mathcal{G}}$.

Further remarks. As for boundary conditions, we assume that $\mathcal{X} \times \mathcal{Z}$ is a compact manifold that contain subregions $\mathcal{X}^{\prime} \times \mathcal{Z}$, with $\mathcal{X}^{\prime}$ corresponding to conformal boundaries, where a subset of the master field components are allowed to blow up; in particular, treating $\mathcal{Z}$ as a compact manifold with non-trivial cycles affects the degrees of freedom that are local on $\partial \mathcal{X}$, as already commented on above. The homogenous Dirichlet boundary condition on $\widehat{\mathbf{P}}$ does not follow from the classical variational principle; instead it follows from the requirement that the field theory BRST operator is a smooth functional differential of a topological field theory $[26,48]$. The latter property is preserved under the addition of topological invariants to $\partial \mathcal{X} \times \mathcal{Z}$. If these contain components of $\widehat{\mathbf{X}}$ in sufficiently high form degree, then they may receive quantum corrections from the $\widehat{\mathbf{P}}^{\star 2}$ and $\widehat{\mathbf{P}}^{\star 3}$ vertices. The topological invariants may thus be non-trivial on-shell, thereby providing boundary micro-state observables appearing in the boundary partition function (as $\widehat{\mathbf{X}}$ is left free to fluctuate at $\partial \mathcal{X} \times \mathcal{Z}$ ); in addition, if the expectation values in $\widehat{\mathbf{X}}$ at $\partial \mathcal{X} \times \mathcal{Z}$ (due to nontrivial cycles and including the zero-form initial data) source forms in $\widehat{\mathbf{X}}$ in higher degrees, then the resulting boundary partition function may contain non-trivial bulk quantum corrections. This suggests that the standard (duality unextended) Chern classes, which only contain one-forms from $A$ and $\widetilde{A}$, correspond to free conformal theories, while their duality extensions, which contain higher forms from $A$ and $\widetilde{A}$, correspond to non-trivial conformal field theories. 


\section{Conclusions}

In this work, we have first presented an alternative to Vasiliev's on-shell formulation of the Type A model in general spacetime dimensions, using the same field content but a different $s p(2)$ gauge symmetry with field independent generators. We have argued that this model propagates the same degrees of freedom as Vasiliev's original equations, and we have provided evidence that the two models are perturbatively equivalent.

Drawing on the field independence of the $s p(2)$ generators of the new model, we have then extended its equations of motion by a dynamical two-form. This extension requires two connection one-forms, gauging the separate left- and right-actions of a complexified higher spin algebra, and a zero- and two-form in opposite (real) bi-fundamental representations. The extended system contains the alternative Type A model as a consistent truncation; at the linerized level, it contains additional degrees of freedom arising in the dynamical two-form, whose analysis we leave for a future work.

Finally, we have proposed that the latter set of equations describes the BRST cohomology of a system that descends from a variational principle, that is obtained by further extension by first-quantized ghosts and an internal graded Frobenius algebra. If this proposal holds true, then these extensions permit the packaging of the equations of motion and the $s p(2)$ gauge conditions, respectively, into a flatness condition and a set of gauge transformations for a single odd superconnetion $\widehat{\mathbf{X}}$. The action also requires the introduction of a supermomentum $\widehat{\mathbf{P}}$ that may quantum deform certain observables, that may be of importance in taking the correspondence between topological open strings and conformal fields beyond the current agreement at the level of conformal particles and free fields [14, 30, 32-34].

Although the extension with dynamical two-form does not retain manifest Lorentz covariance, it is nevertheless suitable for potential extensions of higher spin gravity to more general noncommutative manifolds. Indeed, the extension by the two-form provides a link to topological open string field theory, which is the natural framework for deforming noncommutative geometries.

We have deferred a number of technical aspects for future work: First of all, it remains to map linearized states in lowest weight spaces (particle-like solutions) in $\Phi$ to Fronsdal fields in $W_{\mu}$ by finding a suitable gauge function; for related supporting results for the four-dimensional spinorial formulation, see [49-52]. Furthermore, in order to establish whether the old and the new Type A models are perturbatively equivalent, the first step is to examine whether $K_{i j}^{(\mathrm{diag})}$ and $K_{i j}^{(L)}$ agree in Vasiliev gauge at first sub-leading order.

As for the formulation in terms of the superconnection $\mathbf{X}$, the topology and the boundary conditions of $\mathcal{X} \times \mathcal{Z}$ need to be examined. In particular, $\mathcal{Z}$ needs to contain a non-trivial two-cycle in order for the dynamical two-form to contain the original closed and central element as a non-trivial vacuum expectation value. In this case the alternative Type A master fields arise as a consistent truncation of $\mathbf{X}$; if so, however, the dynamical two-form leads to new local degrees of freedom in spacetime, whose holographic interpretation remains to be given; for related issues in the case of the four-dimensional spinorial theory, see $[10,27]$. 
Our proposal for an action, producing the $s p(2)$ condition as well from a variational principle, relies on the claim made in section 4.3 concerning the BRST cohomology con-

tained in the flat superconnection $\widehat{\mathbf{X}}$ (obtained by extension by first-quantized $s p(2)$ ghosts). In the aforementioned action principle, the $s p(2)$ generators are fixed given operators. In this context, it would be interesting to treat them as new fluctuating degrees of freedom [54-57] of an enlarged string field.

Concerning the basic physical motivation behind our work, namely that from the recent gathering of results concerning the nature of the Noether procedure, it appears that the formulation of higher spin gravity in terms of Fronsdal fields leads to a perturbatively defined quantum effective action making sense in asymptotically maximally symmetric spacetimes, whereas the topological open string field theory formulation provides perturbative expansions around more general backgrounds. In addition, the latter formulation leads to the notion of star product locality, whereby the classical action is built from data obtained from disc amplitudes, thus replacing the more subtle notion of spacetime quasi-(non)locality that needs to be adopted following the standard Noether approach.

Finally, we remark that the alternative $s p(2)$ gauging for the Type A model presented in this work has a direct generalization to the Type B model based on $\operatorname{ssp}(1 \mid 2)$ gauging, whose conformal field theory dual expanded around the anti-de Sitter vacuum consists of free fermions; we hope to present this model in more detail in a forthcoming work.

\section{Acknowledgments}

We have benefited from conversations with N. Boulanger, C. Iazeolla, E. Sezgin and A. Waldron. C.A. is supported by Universidad Andres Bello (UNAB) through a Ph.D. scholarship. R.B. would like to thank the hospitality of UNAB. The work of R.B. is supported by a PDR "Gravity and extensions" from the F.R.S.-FNRS (Belgium). P.S. would like to thank the hospitality of the University of Bologna. The work of P.S. is supported by Fondecyt Regular grants No 1140296 and $N^{\circ} 1151107$ and Conicyt grant DPI 2014-0115.

\section{A Ghost algebra}

Rank one system. Let us consider the four-dimensional graded associative star product algebra $\mathcal{G}$ generated by two Grassmann odd hermitiian operators $(B, C)$ obeying

$$
[B, C]_{\star}=2
$$

and with degree map $\operatorname{deg}_{\mathcal{G}}$, referred to as ghost number, defined by

$$
\operatorname{deg}_{\mathcal{G}}(B, C)=(-1,1) .
$$

The star product algebra can be represented using graded symmetric symbols $f(B, C)$ obeying

$$
B \star f=\left(B+\frac{\partial}{\partial C}\right) f, \quad C \star f=\left(C+\frac{\partial}{\partial B}\right) f .
$$


In this representation, the trace and supertrace operations are given by

$$
\operatorname{Tr}_{\mathcal{G}} f=\left.2 f\right|_{B=0=C}, \quad \operatorname{STr}_{\mathcal{G}} f=-2 \int d B d C f
$$

using standard integration over Grassmann odd variables. Introducing the ghost number operator

$$
w:=\frac{1}{2} C B=-w^{\dagger}, \quad[w, B]_{\star}=-B, \quad[w, C]_{\star}=C,
$$

we define generalized state projectors by

$$
(w-m) \star P_{m \mid n}:=0=: P_{m \mid n} \star(w-n), \quad\left(P_{m \mid n}\right)^{\dagger}=P_{-\bar{n} \mid-\bar{m}},
$$

where $m, n$ are complex numbers; it follows that the left- and right-star multiplication by the ghost number operator has

$$
\operatorname{Spec}\left(l_{w}^{\star}\right)=\operatorname{Spec}\left(r_{w}^{\star}\right)=\left\{ \pm \frac{1}{2}\right\}
$$

and that

$$
\begin{aligned}
P_{-1 / 2 \mid-1 / 2} & =\frac{1}{2}(1+B C), & P_{-1 / 2 \mid+1 / 2} & =\frac{1}{\sqrt{2}} B, \\
P_{+1 / 2 \mid-1 / 2} & =\frac{1}{\sqrt{2}} C, & P_{+1 / 2 \mid+1 / 2} & =\frac{1}{2}(1-B C),
\end{aligned}
$$

that we have normalized such that

$$
P_{m \mid n} \star P_{m^{\prime} \mid n^{\prime}}=\delta_{n, m^{\prime}} P_{m \mid n^{\prime}}
$$

Thus,

$$
\mathrm{Id}_{\mathcal{G}}=\sum_{n= \pm 1 / 2} P_{n \mid n}, \quad w=\frac{1}{2}\left(-P_{-1 / 2 \mid-1 / 2}+P_{+1 / 2 \mid+1 / 2}\right)
$$

and

$$
\operatorname{Tr}_{\mathcal{G}} P_{m \mid n}=\delta_{m, n}, \quad \operatorname{STr}_{\mathcal{G}} P_{m \mid n}=(-1)^{\frac{1}{2}(m+1)} \delta_{m, n}
$$

and hence

$$
\operatorname{Tr}_{\mathcal{G}} f=\operatorname{STr}_{\mathcal{G}} \exp _{\star}\left(\frac{i \pi}{2}(w+1)\right) \star f .
$$

The operators can be represented in a complex vector space $\mathcal{V}$ spanned by

$$
|-1 / 2\rangle=B \star|+1 / 2\rangle, \quad|+1 / 2\rangle=C \star|-1 / 2\rangle,
$$

using labels such that $(w-m) \star|m\rangle=0$, with dual space $\mathcal{V}^{*}$ spanned by

$$
\langle-1 / 2|=\langle+1 / 2| \star C, \quad\langle+1 / 2|=\langle-1 / 2| \star B
$$

defined by

$$
\langle m \mid n\rangle=\delta_{m, n}, \quad m, n= \pm 1 / 2,
$$


using labels such that $\langle m| \star(w-m)=0$; a sesqui-linear form compatible with the reality condition on the star product algebra is defined by the map $\dagger: \mathcal{V} \rightarrow \mathcal{V}^{*}$ given by

$$
(|m\rangle)^{\dagger}=\langle-m|
$$

It follows that the norm of a state $|\psi\rangle=\sum_{m= \pm 1 / 2} \psi_{m}|m\rangle$ is given by

$$
\|\psi\|^{2}=\sum_{m= \pm 1 / 2}\left(\psi_{m}\right)^{*} \psi_{-m}
$$

$\boldsymbol{s p}(\mathbf{2})$ ghosts. The $s p(2)$ ghosts obeying

$$
\left[B_{i j}, C^{k l}\right]_{\star}=2 \delta_{(i}^{k} \delta_{j)}^{l},
$$

generate a 64-dimensional graded associative algebra $\mathcal{G}$ with

$$
\operatorname{deg}_{\mathcal{G}}(B, C):=(-1,1)
$$

and representation in terms of graded symmetric symbols $f$ obeying

$$
B_{i j} \star f=\left(B_{i j}+\frac{\partial}{\partial C^{i j}}\right) f, \quad C_{i j} \star f=\left(C_{i j}+\frac{\partial}{\partial B^{i j}}\right) f .
$$

In this representation, we have

$$
\operatorname{Tr}_{\mathcal{G}} f=\left.8 f\right|_{B=0=C}, \quad \operatorname{STr}_{\mathcal{G}} f=8 \int d^{3} B d^{3} C f .
$$

Introducing the degree operator

$$
w:=\frac{1}{2} C^{i j} B_{i j}=-w^{\dagger}, \quad\left[w, B_{i j}\right]_{\star}=-B_{i j}, \quad\left[w, C_{i j}\right]_{\star}=C_{i j},
$$

we define generalized state projectors by

$$
(w-m) \star P_{m \mid n}:=0=: P_{m \mid n} \star(w-n), \quad\left(P_{m \mid n}\right)^{\dagger}=P_{-\bar{n} \mid-\bar{m}},
$$

where $m, n$ are complex numbers; it follows that

$$
\operatorname{Spec}(w)=\left\{(-3 / 2)^{1},(-1 / 2)^{3},(+1 / 2)^{3},(+3 / 2)^{1}\right\},
$$

where the indices indicate the degeneracy of the eigenvalues. Introducing the $s p(2)$ generators

$$
K_{i j}:=2 i B_{(i}{ }^{k} C_{j) k}, \quad\left[K_{i j}, K_{k l}\right]_{\star}=4 i \epsilon_{(i \mid(k} K_{l) \mid j)},
$$

and letting $f^{(n ; p)}$ denote a symbol obeying

$$
\left(\operatorname{ad}_{w}^{\star}-n\right) f^{(n ; p)}=o
$$


and transforming in the $p$-plet under $\operatorname{ad}_{K_{i j}}^{\star}$, we can decompose $\mathcal{G}$ as follows:

$$
\begin{array}{llll}
P_{-3 / 2 \mid-3 / 2}^{(0 ; 1)} & P_{-3 / 2 \mid-1 / 2}^{(-1 ; 3)} & P_{-3 / 2 \mid+1 / 2}^{(-2 ; 3)} & P_{-3 / 2 \mid+3 / 2}^{(-3 ; 1)} \\
P_{-1 / 2 \mid-3 / 2}^{(1 ; 1)} & P_{-1 / 2 \mid-1 / 2}^{(0 ; 5 \oplus 3 \oplus 1)} & P_{-1 / 2 \mid+1 / 2}^{(-1 ; 5 \oplus 3 \oplus 1)} & P_{-1 / 2 \mid+3 / 2}^{(-2 ; 3)} \\
P_{+1 / 2 \mid-3 / 2}^{(2 ; 1)} & P_{+1 / 2 \mid-1 / 2}^{(1 ; 5 \oplus 3 \oplus 1)} & P_{+1 / 2 \mid+1 / 2}^{(0 ; 5 \oplus 3 \oplus 1)} & P_{+1 / 2 \mid+3 / 2}^{(-1 ; 3)} \\
P_{+3 / 2 \mid-3 / 2}^{(3 ; 1)} & P_{+3 / 2 \mid-1 / 2}^{(2 ; 3)} & P_{+3 / 2 \mid+1 / 2}^{(1 ; 3)} & P_{+3 / 2 \mid+3 / 2}^{(0 ; 1)}
\end{array}
$$

In ghost number zero, there is a four-dimensional space of $s p(2)$ singlets spanned by $w^{n}$, $n=0,1,2,3$, obeying

$$
\begin{aligned}
K_{i j} \star f(w) & =K_{i j}\left(1-\frac{1}{4} \partial_{w}^{2}\right) f(w), \quad K_{i j} w^{2}=K_{i j} w^{3}=0, \\
w \star f(w) & =\left(w+\frac{3}{4} \partial_{w}-\frac{1}{4} w \partial_{w}^{2}\right) f(w) .
\end{aligned}
$$

It follows that the solution to

$$
K_{i j} \star \Delta=0
$$

is given by

$$
\begin{array}{rlrl}
\Delta & =\mu_{+} \Delta_{+}+i \mu_{-} \Delta_{-}=\Delta^{\dagger}, & \mu_{ \pm} & \in \mathbb{R}, \\
\Delta_{+} & =\frac{1}{4}\left(1+2 w^{2}\right), & & \\
\Delta_{-} & =\frac{1}{2}\left(w+\frac{2}{3} w^{3}\right), \\
\Delta_{+} \star \Delta_{+} & =\Delta_{+}, & \Delta_{+} \star \Delta_{-} & =\Delta_{-},
\end{array}
$$

\section{B Trace operations on graded associative algebras}

Given a graded associative algebra $\mathcal{A}$ over $\mathbb{C}$ with product $\star$ and degree map $|\cdot|$, a $\left(\theta_{\mathcal{A}} ; n_{\mathcal{A}}\right)$ trace operation $\operatorname{Tr}_{\mathcal{A}}^{\left(\theta_{\mathcal{A}} ; n_{\mathcal{A}}\right)}: A \rightarrow \mathbb{C}$ obeys

$$
\begin{aligned}
\operatorname{Tr}_{\mathcal{A}}^{\left(\theta_{\mathcal{A}} ; n_{\mathcal{A}}\right)}(a) & =\delta_{|a|, n_{\mathcal{A}}} \operatorname{Tr}_{\mathcal{A}}^{\left(\theta_{\mathcal{A}} ; n_{\mathcal{A}}\right)}(a), \\
\operatorname{Tr}_{\mathcal{A}}^{\left(\theta_{\mathcal{A}} ; n_{\mathcal{A}}\right)}\left(a \star a^{\prime}\right) & =(-1)^{\theta_{\mathcal{A}}|a|\left|a^{\prime}\right|} \operatorname{Tr}_{\mathcal{\mathcal { A }}}^{\left(\theta_{\mathcal{A}} ; n_{\mathcal{A}}\right)}\left(a^{\prime} \star a\right),
\end{aligned}
$$

where the parameter $\theta$ is active $(=1)$ only if $n_{\mathcal{A}} \in 2 \mathbb{Z}$ and the degree map has odd images; we shall refer to a $\left(1 ; n_{\mathcal{A}}\right)$-trace on $\mathcal{A}$ as a supertrace on $\mathcal{A}$, and note that it is cyclic if $n_{\mathcal{A}} \in 2 \mathbb{Z}+1$ or the degree map is even. Given two such algebras, $\mathcal{A}$ and $\mathcal{B}$, their tensor product can be equipped with product and degree maps

$$
(a \otimes b) \star\left(a^{\prime} \otimes b^{\prime}\right):=(-1)^{|b|\left|a^{\prime}\right|}\left(a \star a^{\prime}\right) \otimes\left(b \star b^{\prime}\right), \quad|a \otimes b|:=|a|+|b| .
$$

The composite map

$$
I \equiv \operatorname{Tr}_{\mathcal{A}}^{\left(\theta_{\mathcal{A}} ; n_{\mathcal{A}}\right)} \otimes \operatorname{Tr}_{\mathcal{B}}^{\left(\theta_{\mathcal{B}} ; n_{\mathcal{B}}\right)}: \mathcal{A} \otimes \mathcal{B} \rightarrow \mathbb{C}
$$

defined by

$$
I(a \otimes b):=(-1)^{n_{\mathcal{B}}|a|} \operatorname{Tr}_{\mathcal{A}}^{\left(\theta_{\mathcal{A}} ; n_{\mathcal{A}}\right)}(a) \operatorname{Tr}_{\mathcal{B}}^{\left(\theta_{\mathcal{B}} ; n_{\mathcal{B}}\right)}(b),
$$


has $n_{\mathcal{A} \otimes \mathcal{B}}=n_{\mathcal{A}}+n_{\mathcal{B}}$, and the following cyclicity property:

$$
I\left((a \otimes b) \star\left(a^{\prime} \otimes b^{\prime}\right)\right)=(-1)^{n_{\mathcal{B}}\left(|a|+\left|a^{\prime}\right|\right)+\theta_{\mathcal{A}}|a|\left|a^{\prime}\right|+\theta_{\mathcal{B}}|b|\left|b^{\prime}\right|+|b|\left|a^{\prime}\right|+|a|\left|b^{\prime}\right|} I\left(\left(a^{\prime} \otimes b^{\prime}\right) \star(a \otimes b)\right),
$$

where thus

$$
|a|+\left|a^{\prime}\right|=n_{\mathcal{A}}, \quad|b|+\left|b^{\prime}\right|=n_{\mathcal{B}} .
$$

It follows that

$$
\begin{aligned}
\forall n_{\mathcal{A}}, n_{\mathcal{B}}: & \operatorname{Tr}_{\mathcal{A}}^{\left(1 ; n_{\mathcal{A}}\right)} \otimes \operatorname{Tr}_{\mathcal{B}}^{\left(1 ; n_{\mathcal{B}}\right)}=\operatorname{Tr}_{\mathcal{A} \otimes \mathcal{B}}^{\left(1 ; n_{\mathcal{A}}+n_{\mathcal{B}}\right)} \\
n_{\mathcal{A}}, n_{\mathcal{B}} \in 2 \mathbb{Z}+1: & \operatorname{Tr}_{\mathcal{A}}^{\left(0 ; n_{\mathcal{A}}\right)} \otimes \operatorname{Tr}_{\mathcal{B}}^{\left(0 ; n_{\mathcal{B}}\right)}=\operatorname{Tr}_{\mathcal{A} \otimes \mathcal{B}}^{\left(1 ; n_{\mathcal{A}}+n_{\mathcal{B}}\right)}, \\
n_{\mathcal{A}}, n_{\mathcal{B}} \in 2 \mathbb{Z}: & \operatorname{Tr}_{\mathcal{A}}^{\left(0 ; n_{\mathcal{A}}\right)} \otimes \operatorname{Tr}_{\mathcal{B}}^{\left(0 ; n_{\mathcal{B}}\right)}=\operatorname{Tr}_{\mathcal{A} \otimes \mathcal{B}}^{\left(0 ; n_{\mathcal{A}}+n_{\mathcal{B}}\right)}
\end{aligned}
$$

In particular, the higher spin gravity models use the operations ${ }^{11}$

$$
\operatorname{Tr}_{\mathcal{E}(\mathcal{C}) \otimes \mathcal{F} \otimes \mathcal{G}}^{(1 ; 3(D+1))}(\cdot)=\int_{\mathcal{X} \times \mathcal{Z}} \operatorname{Tr}_{\mathcal{W}} \operatorname{STr}_{\mathcal{F}} \operatorname{STr}_{\mathcal{G}}(\cdot),
$$

in the bulk action, and

$$
\operatorname{Tr}_{\mathcal{E}(\partial \mathcal{C}) \otimes \mathcal{F} \otimes \mathcal{G}}^{(1 ; 3(D+1)-1)}(\cdot)=\int_{\partial \mathcal{X} \times \mathcal{Z}} \operatorname{Tr}_{\mathcal{W}} \operatorname{STr}_{\mathcal{F}} \operatorname{STr}_{\mathcal{G}}(\cdot)
$$

in boundary observables, where

$$
\begin{gathered}
\mathcal{E}(\mathcal{C})=\Omega_{\text {hor }}(\mathcal{C}) \cong \Omega(\mathcal{X} \times \mathcal{Z}) \otimes \mathcal{A} \otimes \mathcal{K} \\
\mathcal{E}(\partial \mathcal{C})=\Omega_{\text {hor }}(\partial \mathcal{C}) \cong \Omega(\partial \mathcal{X} \times \mathcal{Z}) \otimes \mathcal{A} \otimes \mathcal{K}
\end{gathered}
$$

with degree maps given by the form degree on the base manifold $(\mathcal{X} \times \mathcal{Z})$; the $\mathcal{F}=\operatorname{mat}_{2}(\mathbb{C})$ with degree map defined as in (4.32); and $\mathcal{G}$ is the Clifford algebra generated by the $s p(2)$ ghosts with degree map given by the ghost number.

Open Access. This article is distributed under the terms of the Creative Commons Attribution License (CC-BY 4.0), which permits any use, distribution and reproduction in any medium, provided the original author(s) and source are credited.

\section{References}

[1] M.A. Vasiliev, Consistent equation for interacting gauge fields of all spins in (3+1)-dimensions, Phys. Lett. B 243 (1990) 378 [InSPIRE].

[2] M.A. Vasiliev, More on equations of motion for interacting massless fields of all spins in (3+1)-dimensions, Phys. Lett. B 285 (1992) 225 [INSPIRE].

[3] S.F. Prokushkin and M.A. Vasiliev, Higher spin gauge interactions for massive matter fields in 3-D AdS space-time, Nucl. Phys. B 545 (1999) 385 [hep-th/9806236] [INSPIRE].

\footnotetext{
${ }^{11}$ Integration over a manifold $M$ defines a trace operation on $\Omega(M)$ with $\theta=1$, which can be combined with internal supertraces (that are cyclic if the internal algebra has even degree map).
} 
[4] M.A. Vasiliev, Nonlinear equations for symmetric massless higher spin fields in (A)dS(d), Phys. Lett. B 567 (2003) 139 [hep-th/0304049] [INSPIRE].

[5] M.A. Vasiliev, Higher spin gauge theories: Star product and AdS space, hep-th/9910096 [INSPIRE].

[6] X. Bekaert, S. Cnockaert, C. Iazeolla and M.A. Vasiliev, Nonlinear higher spin theories in various dimensions, in Higher spin gauge theories: Proceedings, 1st Solvay Workshop, Brussels, Belgium, 12-14 May, 2004, pp. 132-197 (2004) [hep-th/0503128] [INSPIRE].

[7] X. Bekaert, N. Boulanger and P. Sundell, How higher-spin gravity surpasses the spin two barrier: no-go theorems versus yes-go examples, Rev. Mod. Phys. 84 (2012) 987 [arXiv: 1007.0435] [INSPIRE].

[8] V.E. Didenko and E.D. Skvortsov, Elements of Vasiliev theory, arXiv:1401.2975 [INSPIRE].

[9] E. Sezgin and P. Sundell, Holography in $4 D$ (super) higher spin theories and a test via cubic scalar couplings, JHEP 07 (2005) 044 [hep-th/0305040] [INSPIRE].

[10] N. Boulanger, E. Sezgin and P. Sundell, 4D Higher Spin Gravity with Dynamical Two-Form as a Frobenius-Chern-Simons Gauge Theory, arXiv:1505.04957 [INSPIRE].

[11] E. Witten, Chern-Simons gauge theory as a string theory, Prog. Math. 133 (1995) 637 [hep-th/9207094] [INSPIRE].

[12] M. Bershadsky, S. Cecotti, H. Ooguri and C. Vafa, Kodaira-Spencer theory of gravity and exact results for quantum string amplitudes, Commun. Math. Phys. 165 (1994) 311 [hep-th/9309140] [INSPIRE].

[13] M.R. Gaberdiel and B. Zwiebach, Tensor constructions of open string theories. 1: Foundations, Nucl. Phys. B 505 (1997) 569 [hep-th/9705038] [INSPIRE].

[14] J. Engquist and P. Sundell, Brane partons and singleton strings, Nucl. Phys. B 752 (2006) 206 [hep-th/0508124] [inSPIRE].

[15] C. Arias, N. Boulanger, P. Sundell and A. Torres-Gomez, $2 D$ o-models and differential Poisson algebras, JHEP 08 (2015) 095 [arXiv: 1503.05625] [INSPIRE].

[16] R. Bonezzi, P. Sundell and A. Torres-Gomez, 2D Poisson $\sigma$-models with Gauged Vectorial Supersymmetry, JHEP 08 (2015) 047 [arXiv: 1505.04959] [INSPIRE].

[17] C. Arias, P. Sundell and A. Torres-Gomez, Differential Poisson $\sigma$-models with Extended Supersymmetry, arXiv:1607.00727 [INSPIRE].

[18] B. Sundborg, Stringy gravity, interacting tensionless strings and massless higher spins, Nucl. Phys. Proc. Suppl. 102 (2001) 113 [hep-th/0103247] [INSPIRE].

[19] E. Sezgin and P. Sundell, Massless higher spins and holography, Nucl. Phys. B 644 (2002) 303 [Erratum ibid. B 660 (2003) 403] [hep-th/0205131] [INSPIRE].

[20] I.R. Klebanov and A.M. Polyakov, AdS dual of the critical $O(N)$ vector model, Phys. Lett. B 550 (2002) 213 [hep-th/0210114] [INSPIRE].

[21] N. Boulanger, P. Kessel, E.D. Skvortsov and M. Taronna, Higher spin interactions in four-dimensions: Vasiliev versus Fronsdal, J. Phys. A 49 (2016) 095402 [arXiv:1508.04139] [INSPIRE].

[22] C. Sleight and M. Taronna, Higher-Spin Gauge Theories and Bulk Locality, Phys. Rev. Lett. 121 (2018) 171604 [arXiv:1704.07859] [INSPIRE]. 
[23] M.A. Vasiliev, On the Local Frame in Nonlinear Higher-Spin Equations, JHEP 01 (2018) 062 [arXiv: 1707.03735] [INSPIRE].

[24] E.S. Fradkin and M.A. Vasiliev, Cubic Interaction in Extended Theories of Massless Higher Spin Fields, Nucl. Phys. B 291 (1987) 141 [INSPIRE].

[25] N. Boulanger and P. Sundell, An action principle for Vasiliev's four-dimensional higher-spin gravity, J. Phys. A 44 (2011) 495402 [arXiv:1102.2219] [InSPIRE].

[26] N. Boulanger, N. Colombo and P. Sundell, A minimal BV action for Vasiliev's four-dimensional higher spin gravity, JHEP 10 (2012) 043 [arXiv:1205.3339] [INSPIRE].

[27] R. Bonezzi, N. Boulanger, E. Sezgin and P. Sundell, Frobenius-Chern-Simons gauge theory, J. Phys. A 50 (2017) 055401 [arXiv: 1607.00726] [InSPIRE].

[28] C. Arias et al., Action principles for higher and fractional spin gravities, in Proceedings, International Workshop on Higher Spin Gauge Theories, Singapore, Singapore, November 4-6, 2015, pp. 213-253 (2017) [DOI:10.1142/9789813144101_0012] [arXiv: 1603.04454] [INSPIRE].

[29] E. Sezgin and P. Sundell, An Exact solution of 4-D higher-spin gauge theory, Nucl. Phys. B 762 (2007) 1 [hep-th/0508158] [INSPIRE].

[30] N. Colombo and P. Sundell, Twistor space observables and quasi-amplitudes in $4 D$ higher spin gravity, JHEP 11 (2011) 042 [arXiv:1012.0813] [INSPIRE].

[31] E. Sezgin and P. Sundell, Geometry and Observables in Vasiliev's Higher Spin Gravity, JHEP 07 (2012) 121 [arXiv:1103.2360] [INSPIRE].

[32] N. Colombo and P. Sundell, Higher Spin Gravity Amplitudes From Zero-form Charges, arXiv: 1208.3880 [INSPIRE].

[33] V.E. Didenko and E.D. Skvortsov, Exact higher-spin symmetry in CFT: all correlators in unbroken Vasiliev theory, JHEP 04 (2013) 158 [arXiv:1210.7963] [INSPIRE].

[34] R. Bonezzi, N. Boulanger, D. De Filippi and P. Sundell, Noncommutative Wilson lines in higher-spin theory and correlation functions of conserved currents for free conformal fields, $J$. Phys. A 50 (2017) 475401 [arXiv: 1705.03928] [INSPIRE].

[35] L.F. Alday, D. Gaiotto and Y. Tachikawa, Liouville Correlation Functions from Four-dimensional Gauge Theories, Lett. Math. Phys. 91 (2010) 167 [arXiv:0906.3219] [INSPIRE].

[36] J. Teschner, New Dualities of Supersymmetric Gauge Theories, Springer (2016) [INSPIRE].

[37] R. Aros, C. Iazeolla, J. Noreña, E. Sezgin, P. Sundell and Y. Yin, FRW and domain walls in higher spin gravity, JHEP 03 (2018) 153 [arXiv:1712.02401] [INSPIRE].

[38] D. Quillen, Superconnections and the Chern character, Topology 24 (1985) 89 [InSPIRE].

[39] C. Iazeolla and P. Sundell, Families of exact solutions to Vasiliev's $4 D$ equations with spherical, cylindrical and biaxial symmetry, JHEP 12 (2011) 084 [arXiv:1107.1217] [INSPIRE].

[40] C. Iazeolla, E. Sezgin and P. Sundell, Real forms of complex higher spin field equations and new exact solutions, Nucl. Phys. B 791 (2008) 231 [arXiv:0706.2983] [INSPIRE].

[41] A. Sagnotti, E. Sezgin and P. Sundell, On higher spins with a strong $\operatorname{Sp}(2, R)$ condition, in Higher spin gauge theories: Proceedings, 1st Solvay Workshop, Brussels, Belgium, 12-14 May, 2004, pp. 100-131 (2004) [hep-th/0501156] [INSPIRE]. 
[42] D.J. Gross, A. Hashimoto and N. Itzhaki, Observables of noncommutative gauge theories, Adv. Theor. Math. Phys. 4 (2000) 893 [hep-th/0008075] [INSPIRE].

[43] M. Kontsevich, Deformation quantization of Poisson manifolds. 1., Lett. Math. Phys. 66 (2003) 157 [q-alg/9709040] [INSPIRE].

[44] A.S. Cattaneo and G. Felder, A Path integral approach to the Kontsevich quantization formula, Commun. Math. Phys. 212 (2000) 591 [math/9902090] [INSPIRE].

[45] C.-S. Chu and P.-M. Ho, Poisson algebra of differential forms, Int. J. Mod. Phys. A 12 (1997) 5573 [q-alg/9612031] [INSPIRE].

[46] E.J. Beggs and S. Majid, Semiclassical differential structures, math/0306273.

[47] S. McCurdy and B. Zumino, Covariant Star Product for Exterior Differential Forms on Symplectic Manifolds, AIP Conf. Proc. 1200 (2010) 204 [arXiv:0910.0459] [InSPIRE].

[48] M. Alexandrov, A. Schwarz, O. Zaboronsky and M. Kontsevich, The Geometry of the master equation and topological quantum field theory, Int. J. Mod. Phys. A 12 (1997) 1405 [hep-th/9502010] [INSPIRE].

[49] C. Iazeolla and P. Sundell, Biaxially symmetric solutions to $4 D$ higher-spin gravity, J. Phys. A 46 (2013) 214004 [arXiv:1208.4077] [INSPIRE].

[50] P. Sundell and Y. Yin, New classes of bi-axially symmetric solutions to four-dimensional Vasiliev higher spin gravity, JHEP 01 (2017) 043 [arXiv: 1610.03449] [INSPIRE].

[51] C. Iazeolla and P. Sundell, 4D Higher Spin Black Holes with Nonlinear Scalar Fluctuations, JHEP 10 (2017) 130 [arXiv:1705.06713] [INSPIRE].

[52] D. de Filippi, C. Iazeolla and P. Sundell, From Weyl order to normal order and the central on mass shell theorem in Vasiliev's higher spin gravity, to appear.

[53] M.A. Vasiliev, Star-Product Functions in Higher-Spin Theory and Locality, JHEP 06 (2015) 031 [arXiv: 1502.02271] [INSPIRE].

[54] I. Bars and C. Kounnas, Theories with two times, Phys. Lett. B 402 (1997) 25 [hep-th/9703060] [INSPIRE].

[55] R. Bonezzi, E. Latini and A. Waldron, Gravity, Two Times, Tractors, Weyl Invariance and Six Dimensional Quantum Mechanics, Phys. Rev. D 82 (2010) 064037 [arXiv:1007.1724] [INSPIRE].

[56] R. Bonezzi, O. Corradini and A. Waldron, Is Quantum Gravity a Chern-Simons Theory?, Phys. Rev. D 90 (2014) 084018 [arXiv: 1407.5977] [InSPIRE].

[57] X. Bekaert, M. Grigoriev and E.D. Skvortsov, Higher Spin Extension of Fefferman-Graham Construction, Universe 4 (2018) 17 [arXiv:1710.11463] [INSPIRE]. 